\title{
Paratexte et typographie, métalangage nègre, dans Pigments de Léon-Gontran Damas
}

\author{
Par \\ Mahmoud Elmetwali Attia Ali \\ Professeur adjoint de linguistique- Département du Français \\ Faculté des Lettres- Ismalia
}

DOI: $10.21608 /$ jfpsu.2021.59115.1038 


\section{- Abrégé}

La poésie négro-africaine se caractérise par ses jeux fascinants du paratexte et de la typographie. Elle s'en sert pour mettre en valeur un message codé que le poète nègro-africain veut communiquer. Ce message poétique porte toujours l'empreinte de la particularité de la culture africaine, spécialement la conception de la Négritude.

Dans la poésie négro-africaine, le paratexte et la typographie constituent un métalangage transmettant aux lecteurs des idéologies, des pensées et de toutes les spécificités de l'identité nègre. Les poètes nègro-africains réussissent à utiliser ce métalangage nègre, basé sur le paratexte et la typographie, pour mettre en valeur leur intimité psychologique, leurs émotions, leurs plaintes, leurs douleurs et spécialement leur statut nègre.

La poésie négro-africaine se distingue par la présence martélante de l'Afrique avec ses coutumes, ses aspets rituels et ses entourages idéologiques et culturels. L'africanisation représente, pour les poètes négro-africains une sorte de l'Ego nègre.

Dans cet article, on traite le phénomène de l'axiologie du paratexte et de la typographie dans la poésie et leur rôle pour la trnsmission du message nègre au lecteur, dans Pigments, recueil révolutionnaire de léon-Gontran Damas, un des trois poètes fondateurs de la Négritude. Il se considère comme le plus représentatif de ce mouvement, autrement dit son théoricien.

Pigments est l'un des recueils les plus importants dans la production poétique de la poésie nègro-africaine. Il aborde de nouvelles techniques concernant la nécessité du renouvellement du langage poétique. Il encadre la méthode de Damas pour recompenser l'insuffisance d'expression du langage poétique classique..

\section{- Les mots clés}

Paratexte, Typographie, blanc typographique, majuscule, italique,nègre

\section{- Abréviation}

P: Pigments 


\section{المناص والتشكيل الطبوغرافحى "ميتالغه زنحيه" في ديوان "اصباغ" لليون جونتران داما إعداد}

\section{أ.م.د//محمود المتولى عطيه على}

أستاذ مساعد اللغويات الفرنسية-قسم اللغة الفرنسية

كلية الآداب والعلوم الإنسانية-جامعة قناة السويس

يتميز الثعر الزنجي باستخدامه الرائع للمناص وعتبات النص والتشكيل الطباعي والكاليجرافى كنص موازى للنص الأصلي وكلغة فوقيه او ما وراء اللغة المباشرة. و يستخدها الشاعر الزنجي لتسليط الضوء على رسالة مشفرة يريد الشاعر توصيلها. و تحمل هذه الرسالة الشعرية دائمًا بصمة خصوصية الثقافة الأفريقية ، ولا سيما مفهوم الزنجية. هذا المصطلح ، الذي تم تشكيله من إهانة الزنوج ، اصبح بالنسبة للشعراء الزنوج إكليل فخر ضد إذلال الرجل الأبيض. فهو يسلط الضوء على العناء النفسى عند الزنوج فى البحث عن هويتهم المفقودة. في الثعر الزنجي ، يشكل المناص والطباعة ميتا لغة تنقل للقراء ايديولوجيات وأفكار وكل خصوصيات هوية الزنوج. ويتميز الشعراء الزنوج في استخدام هذه الوسائل التي تشكل اسلوبا لغويا فوقيا عن اللغة التقليدية ، لإبراز علاقتهم النفسية الحميمة ، وعواطفهم ، وشكواهم ، وآلامهم ، وخاصة وضعهم الزنجي. لا يزال الشعر الزنجي الأفريقي منجمًا لا ينضب لم يأخذ حقه ومكانه الذي يستحقه. يتميز بالاندماج بين ثقافتين: الأفريقية و الغربية. يكمن جوهر الابداع في نقطة رئيسية واحدة: تعزيز الثقافة السوداء. ومن هنا جاءت حركة الزنجية. يتميز الشعر الزنجي الأفريقي بالحضور اللافت لأفريقيا بعاداتها وجوانبها الشعائرية وخلفياتها الأيديولوجية والثقافية. ويمثل التمسك بافريقيا ، بالنسبة للشعراء الزنوج ، نوعًا من الأنا الزنجيه و يصبح استحضار إفريقيا فكرة اساسيه عند الشعراء الزنوج. هذا الاستحضار يهيمن على الثعر الزنجي على الرغم من بُعد الوطن الأصلي. إفريقيا وهايتي وجوين وجزر الهند الغربية ومنطقة البحر الكاربي هم من نسل قارتهم الأم: إفريقيا. وهذا يمثل بالنسبة لهم الأنا التي تشكل السلاح المقاوم ضد الظروف السيئة

للزنوج. 
في هذا البحث ، نتعامل مع المناص والتشكيل الطباعي كظاهره فوقيع للغة او ميتالغه وابراز دورها الفعال في توصيل نقل الرسالة الزنجية للقارئ ، بطريقه مشفره وضمنيه من خلال الديوان الثوري "اصباغ" لهاغ للشاعر ليون جونتران داما ، أحد الشعراء الثلاثة المؤسين لحركه الزنوجية. والذى يعتبر الأكثر تمثيلا لهذه الحركة ، وبعبارة أخرى منظّره .

و يعتبر ديوان "أصباغ" من أهم المجموعات الشعرية في الإنتاج الثعر الزنجي حيث يناقش تقنيات

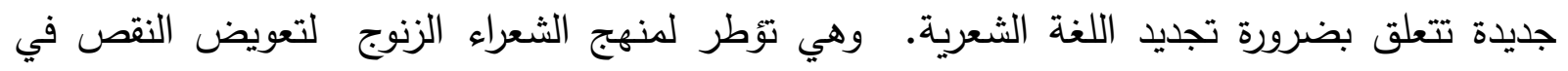
التعبير عن اللغة الشعرية الكلاسيكية. الكلمات المفتاحية

المناص، التشكيل الطبوغرافى، الزنجية 


\section{- Introduction}

La poésie négro-africaine reste encore une mine inépuisable qui n'a pas pris son droit et sa place méritable. Elle se caractérise par la fusion surprenante entre deux cultures: Africaine et occidentale. Le nœud de création réside dans un point principal: la valorisation de la culture noire. D'où naît le mouvement de la Négritude.

La Négritude est un terme forgé et inventé par Aimé Césaire à partir de l'adjectif nègre portant de nombreux signifiés négatifs comme l'oppression, l'exclusion, la colonisation, l'esclavage, la discrimination et l'infériorité avec l'adjonction du suffixe "itude" mettant en évidence l'attitude, la doctrine et la conscience de l'être noir (cf Chanda, 2013).

Ce terme qui, forgé de l'injure nègre, devient pour les poètes négroafricains une couronne de fierté contre l'humiliation de l'homme blanc. Il met en valeur la recherche spirituelle des nègres de leur identité perdue.

L'éclosion de la Négritude a vu la lumière avec trois poètes fondamentaux le sénégalais Léopold Sédar Senghor, le martiniquais Aimé Césaire et le guyanais Léon-Gontran Damas. Les trois poètes ont levé l'emblème de l'Afrique Mère de tous les Noirs. Elle représente l'identité noire avec son entourage culturel, rituel et idéologique dans la poésie des poètes négro-africains.

Les trois poètes ont décidé de proclamer la lutte contre la marginalisation, contre la cruauté et le mépris des Blancs envers les Nègres. Ils abordent ce mouvement littéraire, glorifiant ainsi la suprématie du Noir et de sa culture.

L'évocation de l'Afrique devient une notion de choix chez les poètes négro-africains. Cette évocation domine la poésie nègre malgré l'éloignement du pays natal. l'Afrique, Haïti, la Guyenne, les Antilles, les caraïbes sont descendants de leur Continent Mère: l'Afrique. Celle-ci représente pour eux l'égo formant l'arme résistante face aux mauvaises conditions des Nègres.

Le mouvement de la Négritude représente la révolte du monde opprimé des Nègres contre l'injustice et l'exploitation de la civilisation occidentale: " elle est la définition de tous les damnés de la terre, de tous ceux qui luttent pour la justice politique, sociale et économique, (http://avazooterapia.org.br/journal/kgfil.php?5b3f03=d\% C3\%A9finition-de-lan\%C3\% A9gritude-selon-1\% C3\% A9on-gontran-damas).

On a choisi d'entre les poètes négro-africains Léon-Gontran Damas. Il se considère comme le théoricien de la Négritude. Il est le précurseur des poètes

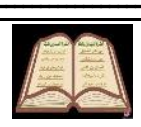

العدى السابع عشر / يناير -2021هـ. (20) 
négro-africains à publier une œuvre poétique que l'on peut considérer comme la Manifestation de ce mouvement.

Damas est le moins connu, bien qu'il soit le plus animé et le plus efficace de la pensée nègre. Il est le plus marginalisé dans le domaine des études sur la Négritude par comparaison avec les autres: Senghor et Césaire.

A l'inverse de Senghor et Césaire qui gardent la supériorité de leur culture nègre mais avec la complémentarité de la culture française, Damas se montre très diffèrent dans sa négritude. On peut même dire extrémiste dans sa conception de Négritude. (cf http://rootsmagazine.fr/leon-gontran-damas-lun-des-peresde-la-negritude/ ). Il appelle les Nègres à retourner à leur histoire, à leur passé, à leur culture et même à leur pays natal. Il veut obliger tout le monde à respecter l'individualité noire et le patrimoine nègre. Il incite vivement les Nègres à défendre leurs droits et à être fiers de leur héritage.

Pour Damas, l'affaire est un combat contre les Blancs au prix du passé odieux de l'exploitation, de la soumission. Il appelle à confronter les pensées méchantes des Blancs envers le Nègre considéré comme l'être le plus opprimé sur la planète. Pour tous les poètes négro-africains, "Damas fut le premier écrivain engagé" (Tshitenge Lubabu M.K. 2012, 1). Il reste fidèle aux valeurs nègres. Il ne demande pas seulement l'égalité mais il proclame le challenge et la supériorité de sa Négritude. Il refuse le mythe que le Nègre est toujours l'équivalent du barbarisme, de l'animalité et du négatif absolu, incapable de vivre sans l'Homme blanc qui peut l'apprivoiser et le soumettre aux règles humaines en raison de sa "mission civilisatrice" (Abiola Irele 2008, 52).

Damas est le premier poète nègre qui prêche la résistance. Il représente pour tous les Nègres la voix absolue de la Négritude. Il représente le cas du nègre fondamental qui reste fidèle à sa Négritude jusqu'à sa mort. Damas incite les poètes nègres à parler une autre langue qui exprime bien leurs âmes, leurs pensées et même leur idéologie. S'ils sont obligés d'utiliser le français, il doit être un français propre aux Nègres, bien dévié du français commun.

On a choisi comme corpus de travail "Pigments", le recueil fondateur et formateur de la pensée nègre. On l'a considéré comme la voix annonciatrice de la Négritude. Il est le reflet de l'esprit révolutionnaire de Damas soit dans le domaine politique soit dans le domaine culturel.

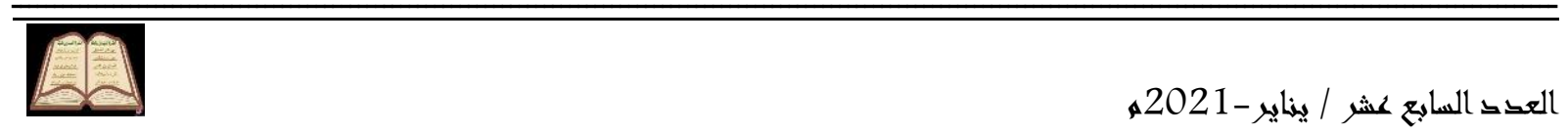


Ce recueil, qui a été publié en 1937, est interdit par la censure en le considérant comme attaque contre la France et une menace de sa tranquillité. Il dessine clairement le pôle révolutionnaire de la vie de Damas

La plupart des études consacrées à la poésie négro-africaine s'intéresse normalement soit au lexique soit à l'oralité. Rares sont les études s'intéressant à la sémiologie de la forme, du paratexte et de la typographie qui représentent un langage révolutionnaire remarquable dans la conscience nègre.

Damas a participé à la Résistance contre le Nazi à cause de sa haine contre le racisme. Il annonce son refus de la persécution des juifs et condamne vivement le drame de "l'holocauste". Il fait appel à la libération des hommes de toutes les forces du mal qui les assujettissent. Dans sa poésie, il attaque violemment toutes les idéologies totalitaires, racistes, politiques, religieuses et sociales du XXe siècle ( $\mathbf{c f}$ Tagore et autres 2011, 143).

Après la guerre, plusieurs poètes ont levé l'emblème du changement et de la révolution contre tous les carcans de l'art poétique traditionnel. Damas a proclamé une esthétique poétique complètement révolutionnaire dans la forme du poème. Il s'est manifesté comme la figure la plus révolutionnaire des poètes nègres du $\mathrm{XX}^{\mathrm{e}}$ siècle.

Le but de notre recherche est d'aborder l'organisation formelle du poème qui s'avère comme un langage parallèle au signe linguistique ou un métalangage implicite. Ce langage spécifique représente pour le poète nègre une universalité de la compréhension totale et éternelle de sa pensée qui " se veut témoignage sur l'homme, témoignage fondé sur l'individualité la plus profonde, la plus universelle " ( Diop, 2001, 12). On essaie de relever la signification profonde de ce langage et d'aborder tous ses traits caractérisant la poésie damassienne s'avérant comme expression de révolte, une "arme de combat", un instrument de libération, bref " un humanisme" ( Mbu-Mputu 2012, 8 ).

Nous y avons recours à l'analyse stylistique paraissant comme la méthode la plus apte et la plus efficace pour aborder la puissance et l'esthétique de la poésie de Damas. Elle se sert de toutes les approches pour mettre en valeur la déviation stylistique du poète figurant sa pensée poétique et valorisant à la fois deux effets: esthétique et communicatif.

Cette recherche a adopté la méthode stylistique de Frédéric Calas et Dominique-Rita Charbonneau mettant en valeur la linguistique textuelle. Cette méthode consiste en premier lieu à valoriser la "représentativité" formelle, c'est- 
à-dire l'étude de la sémiologie de la forme du poème. Elle fait de l'étude du style un processus qui ne sépare jamais le fond de la forme. D'après eux, la saisie de la forme mène à la compréhension du contenu du texte. Chaque élément engendre la compréhension de l'autre en arrivant à recevoir la signification totale dans un ensemble cohérent (cf Calas et Charbonneau 2000, 1).

On a déterminé deux éléments principaux, le paratexte et la typographie qui, d'après la division catégorique de l'analyse formelle de Frédéric Calas et de Dominique-Rita Charbonneau, de nombreux signifiés:

\section{1- La représentativité paratextuelle}

\section{2- La représentativité typographique}

Ces deux éléments précisent l'écart du style du poète de la norme commune de ses contemporains. Cet écart qui "consiste fondamentalement dans les traits du fonctionnement symbolique d'une ouvre permettant de déterminer l'auteur, la période, l'appartenance géographique ou l'école de l'auteur" (Molinié et Chané, 1994, 19).

Dans la poésie négro-africaine, le paratexte et la typographie s'avèrent expressifs et touchants. Ils représentent pour Damas une langue spécifique que les Nègres peuvent le comprendre. Damas annonce la destruction de l'expression classique, fasciste pour l'esprit et pour la création. Il met en valeur un principe très important dans sa poésie: "l'holocauste des mots "(Combe 2014, 12) annonçant le début d'un nouveau code de compréhension, "une cosmogonie de la parole" (Combe 2014, 12) que tous les Nègres puissent comprendre malgré la différence de leurs langues.

Nous aborderons la relation entre l'armature textuelle et la glorification de l'égo nègre et comment cette relation s'enflamme à travers le paratexte et la typographie :

\section{1- La représentativité paratextuelle}

Généralement, le texte poétique s'accompagne de nombreuses informations représentant des seuils pour bien le recevoir et bien découvrir ses profondeurs. Tous les éléments qui entourent le texte servent la bonne présentation du texte au monde et garantissent sa bonne réception.

Selon Gérard Genette, la compréhension des éléments paratextuels participe vivement à valoriser le message poétique. D'après lui, "le paratexte est donc pour nous ce par quoi un texte se fait lire et se propose comme tel à ses lecteurs, et plus généralement au public. Plus que d'une limite ou d'une frontière 
étanche, il s'agit ici d'un seuil ou (......) d'un "vestibule" qui offre à tout chacun la possibilité d'entrer ou de rebrousser chemin" ( Genette 1987,7).

Pour Damas, le paratexte représente une mine inépuisable de signifiés cachés. Il crée dans la poésie une arme clandestine pouvant dire tout que la censure puisse interdire. Il crée un "métatexte" et un "méta-discours"(Labarthe 2004. 191) à travers lequel on peut dire tout ce qui est interdit dans le réel. Le paratexte damassien forme "Le mystère impénétrable, l'inexprimable infini, le néant, la lassitude, le chemin à une compréhension ouverte et infinie" ( Gicquel 1999, 41).

Pour la poésie nègre, le paratexte représente un langage refusant la soumission à la règle rigide de la communication et les formes habituelles du langage traditionnel. Il " permet d'étendre une langue hors pouvoir, dans la splendeur d'une révolution permanente du langage". (Amrit 1995,15$)$. Le poète nègre valorise son engagement et ses conflits à travers le partexte où "les espaces paratextuels" peuvent être vus comme des "micro-discours" ayant valeur programmatique"( Bokiba 2006 P 54).

La poésie traditionnelle compte premièrement sur la lecture orale mais récemment elle a déployé ses efforts pour donner des poèmes à voir mettant en valeur des signifiés spécifiques à travers la tendance de "la poésie visuelle" ou "la poésie figurative" (Radeljković $\mathbf{2 0 1 5}, \mathbf{1})$ où le poème nègre s'excelle à mettre visuellement en évidence les traditions sonores nègres comme les instruments musicaux et les célèbres danses africaines.

Le paratexte met en valeur tous les éléments discursifs hétérogènes que les deux partenaires du recueil, l'auteur et l'éditeur, fonctionnent pour attirer et persuader les lecteurs. Le paratexte représente " une synergie de moyens langagiers qui offre, au sein d'un espace réduit, un condensé des divers problèmes énonciatifs susceptibles de se poser à tout lecteur" (Salbayre, et Vincent-Arnaud 2006, 88).

Pour Léon-Gontran Damas, le paratexte forme le premier lien entre le triangle poète-œuvre-idéologie. La forme paratextuelle de la poésie nègre aborde " un moyen d'accès à une première identification, sous forme de mise en ouvre de présupposés, du texte à venir,..."(Salbayre, et Vincent-Arnaud 2006, 88).

On fait la distinction entre deux sortes de paratexte : le paratexte auctorial qui est complètement sous la responsabilité de l'auteur et le paratexte éditorial qui est partagé entre l'auteur et l'éditeur. Les deux types valorisent une 
singularité et une déviation remarquables du style du poète nègre. Ils travaillent " comme une technique auxiliaire de la stylistique" et cachent "une pointe polémique" (Gicquel 1999, 41).

Commençons par l'analyse du type le plus significatif chez Damas: le paratexte auctorial.

\subsection{Le paratexte auctorial.}

Le paratexte auctorial désigne tous les éléments en rapport avec l'auteur: le nom de l'auteur, les titres et les sous titres, la dédicace, l'épigraphe, la préface, les notes, etc... (cf Genette1987, 14-15). Il représente dans la poésie de Damas un choix bien conscient. Commençons ce type par l'analyse du choix des titres:

\subsubsection{Les titres}

Le titre est le premier moyen d'accès au texte. Le choix du titre représente la première base qui met le poète en contact avec le lecteur. Il introduit directement le lecteur dans le texte. Il se caractérise par sa liberté linguistique et dépasse les règles logiques de la grammaire dans sa composition. On peut le considérer comme" apposition au texte qui la développait. Le texte serait une expansion du titre. Il s'agirait d'une synonymie très spéciale et partiellement implicite, du titre et du texte sur un même axe sémantique" (Rey-Debove 1978, 273).

. Le titre peut valoriser une certaine diachronie en renvoyant aux origines de quelques problèmes et aux idéologies de quelques races ou synchroniques en référant à des événements contemporains et vécus (cf Saminadayar-Perrin 2015,1).

Chez les poètes négro-africains, le titre traduit leur propre culture. Pour Damas, le titre est consciemment choisi. Il met en valeur l'état d'un poète errant, noyé dans ses malheurs, exclu et déraciné de sa terre natale. Il valorise un désir exagéré de retourner au sein de sa patrie: "il chante sa fidélité á l'Afrique, la nostalgie du "pays noir" et son désir de s'abreuver á la source de la sagesse noire" (Huannou 1984, 225).

D'après Gérarad Genette, les titres se divisent en deux grandes catégories: les titres thématiques qui valorisent le contenu ou le thème abordé dans le texte et les titres rhématiques qui donnent des informations sur la nature du texte ou une "désignation générique" de sa formation ou de son courant ( cf Genette 1987, 75-82).

On va commencer l'analyse avec le choix du titre principal du recueil:

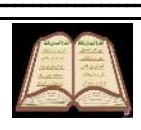




\subsubsection{Le titre du recueil}

Le titre du recueil est bien révélateur de la pensée nègre et de l'acte révolutionnaire chez Damas. Ce recueil, "Pigments", nous présente une figuration du combat des Nègres pour leurs droits.

Selon Larousse, Pigment signifie une "Substance, généralement en poudre fine, insoluble dans les milieux de suspension usuels, utilisée en peinture en raison de ses propriétés optiques, protectrices ou décoratives. (www.larousse.fr , francais , pigment).

Le titre renvoie directement á la couleur de la peau. La couleur noire qui absorbe, selon la pensée du poète, toute couleur et lui donne sa teinte. Il met en valeur l'infériorité qu'éprouvent les Blancs envers les Nègres.

"Pigments" est le premier recueil nègre valorisant non seulement un cri de douleur et d'injustice des Nègres mais soulignant aussi le sublime et la particularité de la culture nègre. Ce recueil évoque la Négritude originelle, celle des origines avant l'arrivée des colonisateurs tout en se concentrant sur la couleur de la peau. Il refuse l'assimilation aux Blancs. D'après lui, le statut nègre n'est pas une honte.

"Pigments" est aussi une révolution dans la poésie nègre en soulignant pour la première fois la fierté d'un poète antillais de la couleur noire de sa peau. "Pigments" vient annoncer la fierté de la Négritude et considérer l'Afrique comme Mère de tous les Nègres du monde (cf Corre 2017, 25).

D'après Damas, ce titre figure bien deux mondes contrariés: l'oppresseur et l'opprimé, la bonté et la méchanceté, la justice et l'injustice. Bref, il met en valeur le conflit Afrique-Europe.

\subsubsection{Les titres des poèmes}

Selon H.Hoek, l'étude du titre doit se faire sur quatre niveaux. Le niveau syntaxique qui paraît quelquefois dévié dans sa structure grammaticale. Le niveau sémantique qui valorise le thème, ses caractéristiques et ses champs lexicaux. Le niveau sémiotique qui figure le titre comme un signe de communication, à la fois informatif et persuasif nous aidant à bien interpréter le texte. Finalement, le niveau pragmatique qui met en valeur tous les éléments culturels, historiques et idéologiques attirant le lecteur (cf H. Hoek 1981, 19-32).

Si l'on passe en revue la classification des titres chez Damas, on trouve que les titres des poèmes valorisent bien l'organisation thématique du contenu suivant deux points logiques : la période et l'expérience du poète. Le titre

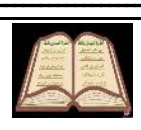

العدى السابع عشر / يناير -2021هـ. (20) 
apparaît donc comme un accord socio-historique "préétabli" qui met en valeur les conditions de la réception (cf H. Hoek 1981, 28).

Dans "Pigments", on rencontre des titres brefs, nominaux sans déterminant, titres non-actualisés. Ce type de titres met en valeur "un langage publicitaire et un langage idéologique, propres à une période déterminée" $(\mathbf{H}$. Hoek 1981, 3). Ce type de titres coïncide avec l'esprit révolutionnaire du poète au début de sa vie s'adaptant avec le temps de la déclaration du mouvement de la Négritude:

\begin{tabular}{|l|l|l|l|}
\hline Poème & Page & Poème & Page \\
\hline Captation & 15 & Hoquet & 35 \\
\hline Obsession & 19 & Solde & 41 \\
\hline Névralgies & 21 & Rappel & 63 \\
\hline Trêve & 23 & Regard & 69 \\
\hline Position & 27 & Réalité & 71 \\
\hline
\end{tabular}

Ce type de titres s'avère identifiant et désignant une certaine idéologie. Il dévoile la pensée et la situation idéologique de la Négritude, il "se trouve dans un rapport paradigmatique avec le texte, dont il constitue un résumé, au moins, partiel: primant l'ensemble du texte, le titre demande à être rapproché de chaque phrase, de l'initiale á la finale" (H. Hoek 1981, 3). Voyons à titre d'exemple:

Captation: Ce titre représente un aspect fondamental dans la poèsie militante de Damas: la figure féminine. Il nous présente à travers ce titre sa captivité par l'odeur de sa femme noire qui, dans la poésie nègre, connote l'Afrique, terre des ancêtres (cf Nkunzimana et autres 2011, 71).

Trêve: signifiant une pause temporaire, évoque les moments heureux qu'on a passés sur la terre natale.

Position: confirme son identité nègre. Il "insiste sur la fermeté de son positionnement idéologique" ( Veté-Congolo 2014, 1).

Hoquet: il met en valeur une forte satire de l'éducation qu'il a recue sous la colonisation et ridiculise la loi de l'assimilation.

Solde: ce titre désigne un refus absolu de l'assimilation aux Blancs en imitant leurs vêtements, leur comportement et leurs modes de vie. Il ridiculise ceux qui vendent leur négritude au nom de la civilisation et s'assimilent aux Blancs.

Il ya aussi des poèmes qui sont liés aux évènements provoquant le passé odieux de la colonisation: 
Regard: invite à jeter des regards contemplatifs sur l'histoire de l'injustice. Le regard conduit à une évocation des mémoires que le poète désigne dans "Rappel".

Rappel: Ce titre valorise la difficulté et l'impossibilité d'effacer les crimes du passé. Ce rappel est obsessionnel.

Obsession: Ce rappel se transforme en une immpossibilité d'oublier le passé sanglant.

Névralgies: cette obsession évoque des vagues névralgiques et des réactions nerveuses en raison de réalité injuste.

Réalité: c'est le titre type valorisant l'état misérable des Nègres devenant l'étendard regroupant tous les damnés de la terre (nègres, juifs et Arabes). La cruauté de la réalité nous conduit à proposer des solutions.

Le titre nominal non-actualisé par des articles ou des déterminants est un titre jouissant de la continuité de "l'universalité et de son rendement idéologique"( H. Hoek 1981, 21). Il s'avère d'une description logique et claire du statut abordé. Il valorise l'attitude spirituelle ou dogmatique. Il parait comme "l'état civil" ( H. Hoek 1981, 64) d'un auteur ou d'un texte. Le titre, ce nominal sans déterminant, est "saisie en intention"(Fromilhague et Sancier-Chateau 1999, 69), c'est-à-dire qu'il valorise une condensation du sens ou de la valeur sémantique ou connotative.

Damas utilise aussi des titres nominaux mais actualisés. On rencontre chez lui un seul titre actualisé par un article défini le vent P.29. Le titre, antéposé d'un déterminant, témoigne de la présence d'une "visée réferencielle" (Fromilhague et Sancier-Chateau 1999, 69), c'est-à-dire la désignation d'une réalité vécue ou imaginaire. Cette référence est la terre natale d'outre-mer avec son climat agité, la Guyane au bord de l'océan.

Il y a aussi des titres actualisés par un adjectif comme "Nuit blanche P.57". Le titre actualisé par un adjectif met en évidence une description minutieuse d'un état ou d'une société et peut mettre en évidence une transformation ( $\mathbf{c f} \mathbf{H}$. Hoek 1981, 76). Ce titre valorise le désir du poète de communiquer avec des Blancs et leur montrer la misère des Nègres. La structure oxymorique du titre valorise le désir de l'accomplissement entre les deux: les Blancs et les Nègres.

Damas reprend la même idée dans le poème intitulé "Blanchi P.59. Le titre de structure participiale décrit le résultat d'une action ou d'une situation, un

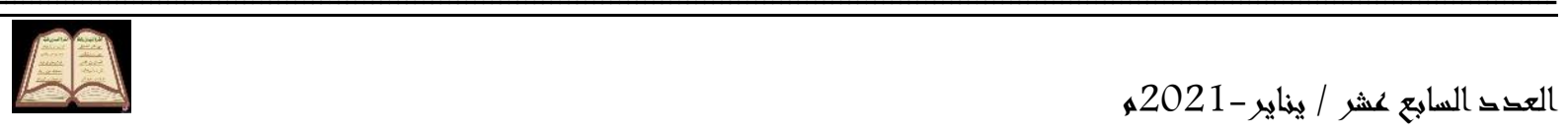


mode non personnel référant à une attitude générale où la précision du temps est absente. Il réfère à "la notion du procès en cours de déroulement" (Lipsky 2003, 71). Ce titre met en valeur l'effort de la mère de faire de son enfant nègre un enfant blanchi, c'est- à-dire un enfant noir vivant comme les enfants des Blancs.

Mais Damas dénonce le désir des mères de faire de leurs enfants nègres des enfants blanchis en réhabilitant la légende de la Négritude dans Pareille à ma légende P.61, une dénonciation de la pensée des Européens sur le nègre et aborde un enchantement de la sagesse et de la culture noires.

Damas utilise aussi des titres adverbiaux qui donnent des indications sur le temps. Le titre Si souvent P.49 décrit les malheurs des Nègres et le sentiment de peur qui domine le Nègre en raison de sa race tandis que Bientôt P.55 connote, pour les $\mathrm{N}$ ègres, un proche avenir heureux plein de chant et de danse.

Le poète donne aussi des titres qui se composent de "syntagmes prépositionnels" (H.Hoek 1981, 74), des titres de structure prépositionnelle, ajoutant une détermination ou une précision circonstantielle : En file indienne P.31, qui décrit l'invasion des Français des Antilles et l'expression décrit les massacres déroulés les uns après les autres.

Dans son attente P.33, ce titre valorise l'effroi, le tourment des Nègres et leur attente déçue et pénible, Pour sûr P.53 dénote une affirmation de sa haine et met en valeur une plainte d'un mal insupportable, Sur une carte postale P.77, connote l'inscription de tous les lieux de son enfance qui paraissent comme "des coins du paradis" (cf Gyssels 2017, 3).

Le titre peut aussi valoriser une abréviation, S.O.S P.51 renvoyant au système international de sécurité. Il s'en sert pour deux raisons : un renforcement du message envoyé par un nègre et une mise en comparaison de deux injustices: Nègres / Blancs et Juifs /Allemands (cf https://www.etudier.com/dissertations/Analyse-De-s-0-s-Damas/50841632.html ).

Le titre peut marquer un contexte historique et culturel. Il peut valoriser une "historicité" ou " un processus culturel" ( H. Hoek 1981, 26-27". Ce type paraît avec des titres qui deviennent des archives conservatrices d'une situation historique et idéologique. On le rencontre dans Et cætera P.79, un adverbe qui dénonce l'envoi des Combattants Sénégalais à la participation dans la Guerre mondiale et montre comment les Français les mettent devant eux sur le Front 
pour recevoir les balles des Allemands. On décrit cette crime comme une "Honte Noire" ( Kesteloot 2004, 121).

Le cas est le même avec Shine P. 65, titre anglais, pour honorer le monde du jazz que le poète considère comme étant un monde spécifique des Nègres. Cet entourage jazzique se complète avec ce titre Des billes pour la roulette $\mathbf{P}$. 75 valorisant l'atmosphère des bars. Ce titre met en valeur une comparaison implicite entre le mouvement de la roulette et le destin du Nègre.

La même idée se répète avec Limbé $\mathbf{P . 4 3}$, un nom donné à une sorte de poupée noire pour les enfants nègres. Les enfants blancs ont beaucoup aimé. Cette poupée noire évoque le pays natal et met en valeur une enfance déracinée ou kidnappée. Elle souligne l'injustice des colonisateurs qui volent même l'enfance (cf Moudileno 1997, 182).

Damas emploie rarement des titres longs. Ce titre, "argumentum" (H.Hoek 1981, 13), c'est-à-dire de nature argumentative, annonçant le contenu du poème. Il présente des prétextes renforçant le point de vue du poète: Ils sont venus ce soir 13, ce titre est l'un des rares cas qui est construit d'une phrase complète figurant la catastrophe de l'arrivée des Blancs aux colonies et les crimes qui ont accompagné leur arrivée.

Le même type se répète avec Un clochard m'a demandé dix sous p.39 qui met en valeur le vagabondage du nègre et son errance dans les rues.

Un autre poème portant le titre Ils ont $\mathbf{P}$. 73, cette phrase incomplète, avec elliptique du complément, aborde une nouvelle vision de la race nègre basée sur la fierté, une vision qui conduit le lecteur à deviner des suppositions. Ce titre sans complément accentue une comparaison entre les caractères que les Blancs et les Noirs possèdent. Il laisse les guillemets ouverts pour mettre le lecteur en suspense. Le complément supprimé est une arme de dire implicitement tout ce que la censure peut refuser.

On rencontre aussi le titre narratif Il est des nuits $\mathbf{P . 2 5}$ que le poète utilise pour raconter un conte merveilleux ou un récit imaginaire décrivant sa nostalgie au paradis de la patrie.

Dans "Pigments", on distingue aussi des titres "adnominal" (H. Hoek 1981, 76), c'est-à-dire composés d'un syntagme nominal unifié par la préposition "de" et jouent le rôle complément déterminatif. Ce type se considère comme "l'archimodèle logico-sémantique" (cf H. Hoek 1981, 76-77) dans l'explicitation du titre. Ce type paraît clairement avec le titre "La complainte du 
nègre P.47" valorisant à l'extrême la misère des nègres et spécifiant leur peine psychologique et corporelle.

Chez Damas, le choix des titres est bien significatif et bien mis en valeur pour souligner les problèmes majeurs de la vie des Nègres. A travers la série des titres des poèmes, Damas valorise tous les maux des Nègres, leurs souffrances, leurs déceptions et leurs ambitions. Les titres se présentent comme des "signes doubles" (Riffaterre 1983, 130) où ils soulignent le statut nègre individuel concrétisant un cas spécifique du poète mais croisé avec l'idéologie commune des Nègres. Le poète se transforme du poète nègre à l'Homme nègre qui devient la voix de l'identité nègre et l'emblème de tous les Nègres du monde: africains, caraïbes et Américains.

Grâce à l'arrangement des titres dans "Pigments", on a des archives historiques qui conservent toutes les agressions contre les Nègres. L'organisation de la titrologie dans le recueil affirme l'unité de la signification et attire l'attention du lecteur sur une idéologie et une pensée bien précises. Il commence avec un poème " Ils sont venus ce soir" mettant en valeur l'horreur qui accompagne l'arrivée des Blancs en Afrique et se termine par le poème " et Cætera" dénonçant l'envoi des Combattants Sénégalais pour aider la France contre le Nazi. Ce début et cette fin de "Pigments" met en évidence une comparaison entre la situation de la France avec les Africains et celle de l'Afrique pendant la Guerre.

Les titres de "Pigments" abordent des remèdes pour les maux des Nègres et leur présentent un savoir-vivre dans l'univers injuste. Le recueil réclame la liberté. Il élève l'emblème de la fierté de la race nègre. Il valorise une insistance sur "l'identité pigmentée...et l'expérience vécue du Noir" (Gyssels 2010, 4).

\subsubsection{La dédicace}

Généralement, la dédicace désigne "un Hommage qu'un auteur fait de son œuvre à quelqu'un en la lui dédiant par une mention imprimée en tête du livre" (www.larousse.fr > francais > dédicace). Sa fonction est premièrement de mettre en contact l'auteur et le dédicataire. La dédicace traduit une certaine évolution dans la démarche spirituelle de l'auteur et une vue d'ensemble sur ses attitudes idéologiques.

Dans la poésie nègre, la dédicace porte une méditation spécifique et personnelle soit sur le niveau culturel, soit sur le niveau idéologique ou sur le statut politique. La dédicace résume les efforts que les partisans de la Négritude

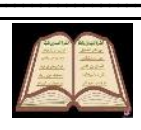

العدى السابع لشر / يناير -2021هـ. 
ont déployés.La dédicace met en valeur l'engagement dans la défense de l'identité nègre (cf Alvina Ruprecht, (cf Taiana et Ruprecht Alvina, 1995, 220). Le tableau suivant expose les dédicataires dans les poèmes de "Pigments":leurs attitudes, leur lutte, leur soutien et leur participation au champ de la Négritude.

Tableaux des Dédicataires

\begin{tabular}{|c|c|c|c|}
\hline poème & $\begin{array}{c}P a g \\
e\end{array}$ & $\overline{\text { Dédicace }}$ & Nom Propre \\
\hline Ils sont venus ce soir & 13 & Léopold-Sédar Senghor & $\begin{array}{l}\text { Un des trois poètes fondateurs de la } \\
\text { Négritude. il se considère comme } \\
\text { une des bases de la Francophonie } \\
\text { (cf Constant et Kahiudi 2009, 11) }\end{array}$ \\
\hline A la mémoire de G.M & 17 & G.M & $\begin{array}{l}\text { Cette abréviation désigne } \\
\text { Géorges Mizaine, un des } \\
\text { amis très proches de } \\
\text { Damas. (cf Poujols 2oo3, } \\
\text { 166). }\end{array}$ \\
\hline Il est des nuits & 25 & Alejo Carpentier & $\begin{array}{l}\text { Intellectuel et artiste des } \\
\text { Caraïbes. Il est l'un des } \\
\text { défenseurs solides de son } \\
\text { identité nègre (cf Chancé } \\
\text { 2001, 6) }\end{array}$ \\
\hline Position & 27 & $J . D$ & $\begin{array}{l}\text { J.D est une abréviation d'une } \\
\text { personne dont l'identité est } \\
\text { inconnue. Son identité et son } \\
\text { idéologie sont bien conservées dans } \\
\text { la mentalité du poète. (Dehoux } \\
\mathbf{2 0 1 8 , 2 2 0 )}\end{array}$ \\
\hline Le vent & 29 & $\begin{array}{l}\text { Henriette et Jean-Louis } \\
\text { Baghio'o }\end{array}$ & $\begin{array}{l}\text { Un des premiers nègres intitulé du } \\
\text { magistère. Henriette est sa petite } \\
\text { fille morte dans un accident. (cf } \mathbf{W} \text {. } \\
\text { Scheel, 2017, 1). }\end{array}$ \\
\hline Hoquet & 35 & Vashti et Mercer Cook & $\begin{array}{l}\text { Il est le premier américain d'origine } \\
\text { africaine qui occupe un poste } \\
\text { d'ambassadeur en Gambie puis au }\end{array}$ \\
\hline
\end{tabular}




\begin{tabular}{|c|c|c|c|}
\hline & & & 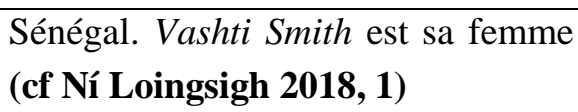 \\
\hline Solde & 41 & Aimé Césaire & $\begin{array}{l}\text { Aimé Césaire est le troisième } \\
\text { chevalier de la Négritude. C'est lui } \\
\text { qui a forgé le terme Négritude. Il } \\
\text { représente pour les Nègres le } \\
\text { symbole de la nouveauté. (cf . } \\
\text { Abiola Irele 2008, 161). }\end{array}$ \\
\hline Limbé & 43 & Robert Romain & $\begin{array}{l}\text { Membre actif de la défense des } \\
\text { droits des Nègres. IL a soutenu } \\
\text { fortement la publication de Pigments } \\
\text { (cf Macé 2008,1) }\end{array}$ \\
\hline La complainte du nègre & 47 & Robert Goffin & $\begin{array}{l}\text { Un avocat belge, auteuret et poète, } \\
\text { le premier qui a écrit un livre sur le } \\
\text { monde du jazz. Il est un des grands } \\
\text { partisans de la Négritude (cf } \\
\text { Thomas et Lecomte 1983, 191) }\end{array}$ \\
\hline Nuit blanche & 57 & Sonia et Georges Gavarry & $\begin{array}{l}\text { Georges Gavarry est l'un de grands } \\
\text { partisans de la rencontre France- } \\
\text { Afrique. Lui, et sa Femme Sonia, } \\
\text { ont construit un club où chaque } \\
\text { semaine se déroulent les discussions } \\
\text { sur ce sujet. (cf Biasini 1995, 144). }\end{array}$ \\
\hline Blanchi & 59 & Christiane et Alioune Diop & 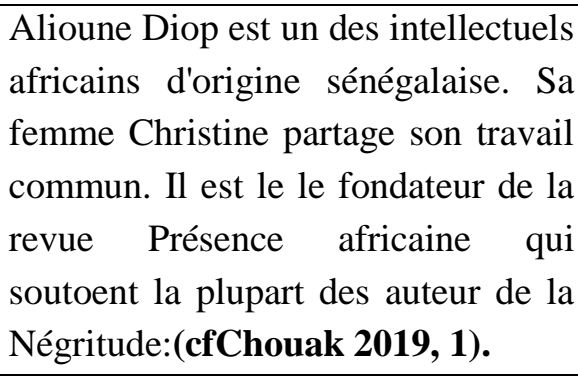 \\
\hline Rappel & 63 & Richard Danglemont & $\begin{array}{l}\text { Un intellectuel antillais qui met en } \\
\text { valeur le rapprochement entre } \\
\text { l'Afrique et les Antilles. Il considère } \\
\text { l'Afrique comme la patrie Mère (cf } \\
\text { Manchuelle 1992, 376) }\end{array}$ \\
\hline Shine & 65 & Louis Armstrong & $\begin{array}{l}\text { Il est musicien de jazz. C'est lui qui } \\
\text { a inventé le rythme jazzique. Il } \\
\text { contribue vivement à répandre la }\end{array}$ \\
\hline
\end{tabular}




\begin{tabular}{|l|r|l|l|}
\hline & & & $\begin{array}{l}\text { culture des Noirs américains, (cf } \\
\text { Http://baamadou.overblog.fr/) }\end{array}$ \\
\hline Savoir-vivre & $\mathbf{6 7}$ & Etienne Zabulon & $\begin{array}{l}\text { Activiste et militant des droits des } \\
\text { nègres (cf Macé 2008,1). }\end{array}$ \\
\hline Regard & $\mathbf{6 9}$ & Jacques Howlett & $\begin{array}{l}\text { Il est Africaniste. Il est un des } \\
\text { fondateurs avec Alioune Diop de la } \\
\text { revue Présence Africaine (cf } \\
\text { data.bnf.fr }>\text { jacques_howlett) }\end{array}$ \\
\hline
\end{tabular}

La dédicace représente un leitmotiv constant du style de Damas. Elle représente implicitement le porte-parole de l'idéologie et de l'identité nègre. Le dédicataire, à travers son engagement, représente un miroir de l'égo nègre et de la liberté de l'Afrique. (cf Dieng 2009, 2). Chez Damas, la dédicace souligne de nombreuses fonctions. Elle devient un témoignage pour l'histoire. Cela 'affirme avec la compréhension de trois éléments: le dédicataire, le contexte et l'occasion. Elle peut marquer:

\subsubsection{Une attaque}

Le poème Solde est dédié à Aimé Césaire. Ce poème est une forte dénonciation de décret de la départementalisation considérant les anciennes colonies comme des départements français. La dédicace met en évidence la colère du poète de son ami Césaire après son vote d'accord sur ce décret. Damas l'accuse d'être partenaire du complice ( cf Malela Buata, 2008, 171).

\subsubsection{Un reproche}

Le poème "Ils sont venus ce soir", dédié à Léopold-Sédar Senghor, porte un reproche poli de Damas à son ami de combat après avoir accepté l'envoi des combattants Sénégalais pour soutenir la France contre le Nazi.

\subsubsection{Un souhait}

Dans Position, Damas dédie le poème à J.D, une personne inconnue, réelle ou imaginaire (cf Dehoux 2018, 220). Le poète désigne à travers une contemplation créatrice du mouvement du clavier du piano où les touches noires se côtoient avec les touches blanches, l'accomplissement entre les deux est indispensable pour réaliser de plus beaux morceaux musicaux. Le cas est le même pour l'harmonistion Noirs - Blancs. 


\subsubsection{Une glorification}

La dédicace met en valeur une sorte de fierté de ce que le Nègre peut réaliser et le met en compétition avec le Blanc. Elle honore des Nègres à être les premiers dans leur domaine:

Mercer Cook, premier ambassadeur américain d'origine africaine. Alioune Diop, premier nègre qui a fondé une maison d'édition. Louis Armstrong, l'inventeur du jazz qui devient la figuration audible de la culture et du folklore africaines.

Jean-Louis Baghio'o, un des premiers nègres qui a obtenu un magistère.

\subsubsection{Une reconnaissance}

Dans "Pigments", la plupart des dédicaces valorisent le rôle de quelques personnes dans le mouvement de la Négritude. Ils sont des amis comme Géorges Mizaine, des activistes nègres comme Etienne Zabulon, Richard Danglemont , Robert Romain et Alejo Carpentier, des Blancs partisans des droits des Nègres comme Jacques Howlett, Georges Gavarry , Robert Goffin et des femmes activistes dans le domaine social comme Vashti, Sonia et Christine. Du point de vue linguistique, le nom propre dédicataire ajoute de ses caractères personnels au sens large de l'idéologie collective, au signifié du poème et à la pensée du poète, ce qu'on appelle par "l'hypersémanticité" ( Leroy 2004, 121) idéologique et poétique.

\subsubsection{La préface}

Normalement, la préface signifie "un discours placé en tête d'un livre, pour en faire connaître les vues ou le plan, prévenir des objections ou répondre à des critiques" (www.espacefrancais.com > la-preface). Deux éléments déterminent l'importance et la valeur de la fonction préfacielle: le contenu et le préfacier.

Selon Gérard Genette, il y a deux types de préface : la préface auctoriale originale simultanée avec l'apparition de la première édition et la préface tardive qui s'ajoute après. La préface originale rend deux fonctions essentielles: garantir une bonne lecture et présenter une vision claire de l'idéologie et de la pensée de l'auteur (cf Genette 1987, 182-183).

La préface originale de Pigments est écrite par Robert Desnos qui est un célèbre poète surréaliste. Il se caractérise par son engagement politique et idéologique. Dès la déclaration du Surréalisme, ce mouvement soutient tous les 
mouvements révolutionnaires. Il favorise l'encouragement et le refuge pour la Négritude. Les deux mouvements se rapprochent car leurs principes sont presque les mêmes: l'appel à la libération de l'esprit, la domination de l'inconscience et du rêve, la nouveauté dans l'art poétique et la découverte de nouveaux chemins pour la création littéraire.

La préface de "Pigments" représente la première Manifeste de la Négritude. Le lecteur et le critique peuvent s'en servir comme "voie d'accès" (Bokiba 2006, 54) à l'œuvre et valoriser la composante idéologique de l'auteur. Elle met en évidence l'intimité du poète nègre et révèle ses ambitions. Elle constitue d'avance un jugement sur la poétique du poète. Elle est annonciatrice du contenu du recueil et révélatrice de l'esprit révolutionnaire de Damas.

Dans la preface, Desnos aborde le statut révolté de Damas: "le poète nègre fier d'être nègre" ( Damas 2003, 10). Il présente Damas comme le poètehéros de la race nègre et un des premiers qui ont appelé l'union des Nègres de tout le monde. Celui-ci montre que l'adhésion de tous les Nègres est inévitable car la mère est l'Afrique.

Dans la poésie négro-africaine, la préface souligne une "légitimation littéraire" (Bokiba 2006 P 54) de la ligne militante de la poésie nègre. Desnos proclame dans La préface que Damas insiste sur le moi et le non-moi, sur la nécessité de la coexistence de deux cultures, sur la légitimé de la différenciation. Il annonce que Damas est Nègre et qu'il tient au maximum sa Négritude. ( cf Mansfield 2006, 243).

\subsubsection{La postface}

La postface désigne "un texte ajouté à la fin d'un livre ou d'un écrit en guise de supplément ou de conclusion, généralement pour émettre un commentaire, une explication ou un avertissement" (www.cnrtl.fr > definition > postface).

L'édition originale de "Pigments" paraît sans postface. Dans cette édition, on voit la postface présentée par Sandrine Pujols, spécialiste de l'étude de la production poétique de Damas comme elle est la préfacière de son célèbre recueil Black Label.

La postface paraît avec la publication de la dernière version réunissant "Névralgies", son dernier recueil et "Pigments", son premier recueil. Elle concrétise le modèle du résistant nègre qui cherche sa Négritude partout. Tous les deux recueils dessinent clairement les deux pôles de la vie de Damas: poète

$$
\text { العقد السابع عشر / يناير - 2021هـ. (20) }
$$


résistant, combattant pour sa Négritude, novateur et conservateur de ses doctrines. Cette postface est le reflet de ces caractéristiques personnelles: "la poésie de Damas est névralgique, voire névrosée" ( Corre 2017, 27).

La postface met en relief la mémoire obsessionnelle qui suit le poète de ses débuts jusqu'à sa mort et il ne peut jamais l'oublier. De temps en temps, le passé laid et honteux de l'homme blanc rayonne en lui rappelant toutes les scandales que les Européens ont commis. Cette mémoire qu'il dénote par l'expression "la tyrannie de la mémoire" (Talahite-Moodley 2007, 181) lui présente les prétextes de l'intolérance ou de l'oubli de ces crimes corporels et psychologiques.

La postface et la préface encadrent une ouverture sur l'œuvre, un élargissement avec de nouveaux horizons en abordant de nouvelles visions poétiques. Les deux sont communicatives : la préface valorise une présupposition tandis que la postface met en valeur une finalité.

Toutes les composantes du paratexte auctoriale se communiquent avec celles de l'édition pour aborder des seuils ouvrant de nouveaux horizons pour la compréhension de la poétique de Damas.

\section{2 le paratexte éditorial}

Le paratexte éditorial désigne tout ce qui est sous la responsabilité directe de l'éditeur, mais en participation et sous la surveillance de l'auteur. Généralement, il s'agit toujours de la forme extérieure du livre.

L'analyse du paratexte éditorial s'appuie sur deux côtés: description et argumentation. Il rend un rôle instructif sur la nature des textes et figure une vision présupposée du dedans. Ce type de paratexte est souvent organisé pour susciter le lecteur au livre de sa forme et son organisation extérieures. De ce point, le paratexte éditorial s'avère comme une "spécificité linguistique et communicationnelle" (cf Lane 1991,92).

\subsubsection{La couverture}

L'analyse de l'image de la couverture d'un recueil représente une présupposition explicative de son contenu. La couverture est en relation directe avec les textes inclus. La compréhension des indices extérieurs de la couverture nous présente un " code herméneutique" (Ribière, M. et Baeten, J. 1994, 89) du statut révolutionnaire du poète nègre.

L'image de la couverture aborde un code spécifique de compréhension, un propre langage entre le poète et le lecteur. La cohérence et la complémentarité du 
système visuel des signes et des éléments sémiologiques sur la couverture jouent un rôle essentiel pour faire comprendre l'objectif poétique du poète et tracent le chemin d'une lecture consciente.

La couverture originelle de "Pigments" montre clairement des slogans dans la vie des Nègres et figure l'image du nègre révolté et perdu entre le déracinement et l'insistance sur ses origines.

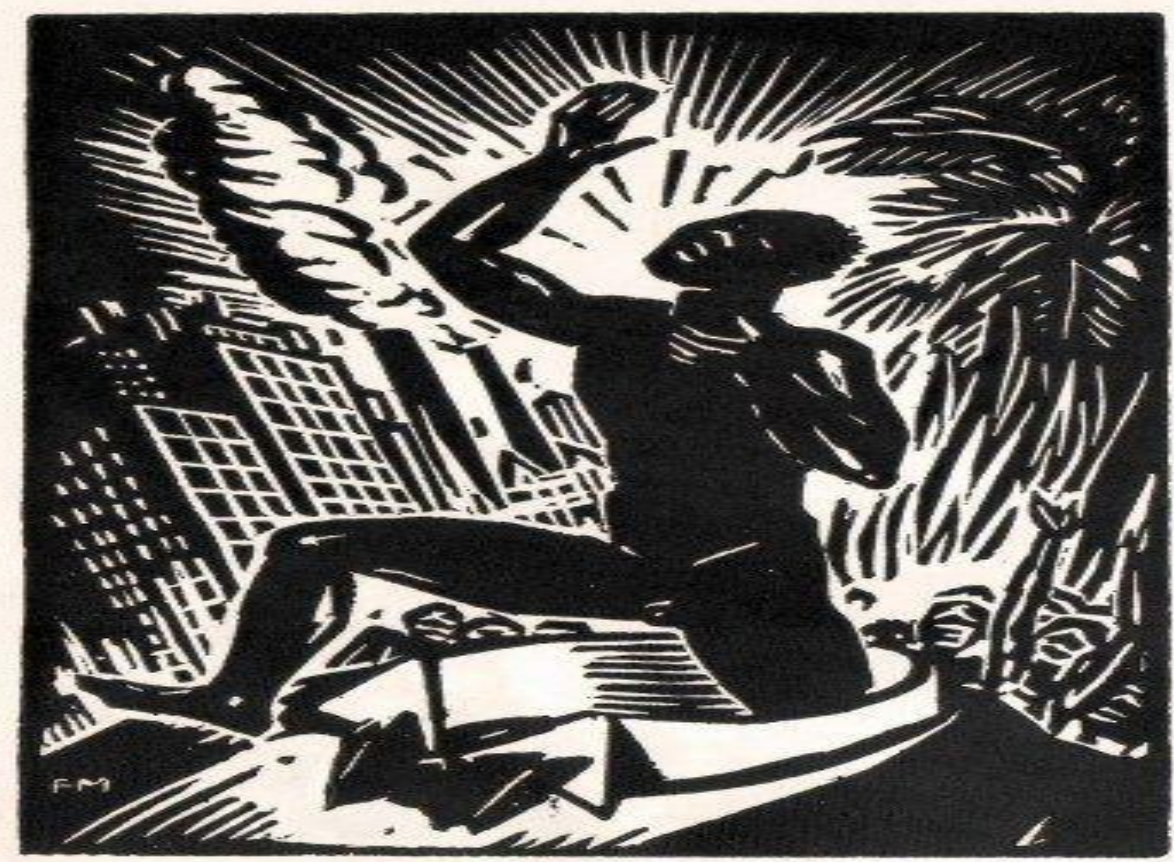

En passant par la description formelle de la couverture, elle est une image de gravure sur bois créé par le belge Frans Masereel. Il adopte le style expressionniste des artistes allemands qui expriment, par cet art, leur refus et leur confrontation du Nazi (cf Bergeret 2007, 2).

Ce type de gravure est un outil d'expression de malaise, d'oppression, d'injustice et de révolte. Il paraît dès la fin de la Première Guerre Mondiale et prend son essor avec la montée du Nazisme. Il met en valeur à travers "le message photographique" ou la "rhétorique de l'image" (Véron, 1994, 45) de nouvelles suppositions pour une meilleure vie.

La gravure sur le bois ou la "xylogravure" met en valeur l'esprit révolutionnaire dominant ce temps: anti-colonialisme, anti-esclavage, antinazisme et antifascisme. Elle valorise les réactions psychanalytiques résultant 
des visions d'angoisse, de malaise et de menace des guerres et des systèmes totalitaires ( cf fr.wikipedia.org > wiki > Gravure_su..).

Pour les poètes nègres, cette forme révolutionnaire de gravure représente la forme idéale de l'expression de leur statut. La gravure sur le bois s'appuie essentiellement sur deux couleurs: le blanc et le noir(cf Milliot, 1995, 69-70). Ce sont les deux couleurs qui existent sur la couverture. Ces deux couleurs valorisent clairement le conflit discriminatoire. Elles inspirent au lecteur des réactions et des motivations présupposées du contenu du recueil.

Ces deux couleurs évoquent l'aspect conflictuel entre deux mondes: "un signe de base qui contient en germe tout le combat" ( Barthes 1957, 16). Elles représentent une confirmation sur la position idéologique du poète et son refus de la loi de l'assimilation imposée sur les Nègres pour effacer leur culture. La majorité de la couverture est en noir pour mettre en évidence la suprématie raciale de la Négritude.

La couverture présente à travers l'alternance noir/ blanc deux possibilités : l'opposition et la la complémentarité. L'opposition se dévoile par le rattachement du Nègre à ses origines et la complémentarité par le besoin frappant de la différenciation et de l'existence de l'autre. La couverture communique implicitement au lecteur trois messages :

\subsubsection{L'espace}

La couverture évoque deux espaces contrariés : d'un côté, les hauts immeubles, un signe du monde blanc avec son luxe et de l'autre côté, les arbres, signe représentatif des territoires de l'Afrique et de son pays natal aux Caraïbes.

\subsubsection{Le corps}

Le corps est celui d'un jeune nègre, fort et bien musclé. Il existe quelques points blancs sur sa peau résultant de l'imposition de l'assimilation. Mais il résiste solidement.

\subsubsection{Les vêtements}

La couverture aborde l'apparence vestimentaire de l'homme blanc: une veste noire, une chemise blanche et un nœud papillon. Cette apparenc valorise l'entourage raffiné de l'homme occidental blanc: chic, civilisé et puissant par sa culture. Mais l'élégance vestimentaire ne s'accomplit que par la différence et l'harmonie des deux couleurs : chemise blanche et veste noire.

Les trois signes, l'espce, le corps et les vêtements, évoquent l'aspect conflictuel entre deux civilisations. Le corps du nègre se libère de tous les 
carcans de l'assimilation et évoque le mythe du retour au paradis de ses rêves: la Terre-Mère. Le nègre nu représente l'émancipation noire de ses vêtements occidentaux et le débarrassement d'eux comme il sort d'un cratère du volcan (cf Bergeret 2007, 2). Sur la couverture, figure un halo de lumière représentant l'explosion de la colère nègre. Une colère qui s'accumule à cause del' injustice et de l'oppression des décennies d'années.

Le nègre nu se débarrassant de ses vêtements représente une des visions de la Négritude: " Un jour, le Nègre s'empara de la cravate du Blanc, se saisit d'un chapeau melon, s'en affubla, et partit en riant..." (www.cairn.info > revue-lestemps-m). L'image du Nègre tout puissant et musclé évoque l'image du héros légendaire chez les anciens Grecs, Héraclès abordant l'image du"héros civilisateur" (Mactoux et Geny 1990, 155) qui est fondateur de la ville et enseignant de bonnes sciences aux hommes. Cette figuration concrétise le modèle du Nègre révolté qui insiste sur le rattachement à ses origines. Elle met en évidence une "représentation prototypique " (Ribière, M. et Baeten, J. 1994, 81) du héros, c'est- à-dire une figuration idéale dans la mentalité collective de tous les Nègres du monde.

Mais la dernière édition qui réunit Pigments et Névralgies paraît avec une couverture calme, simple et unie:

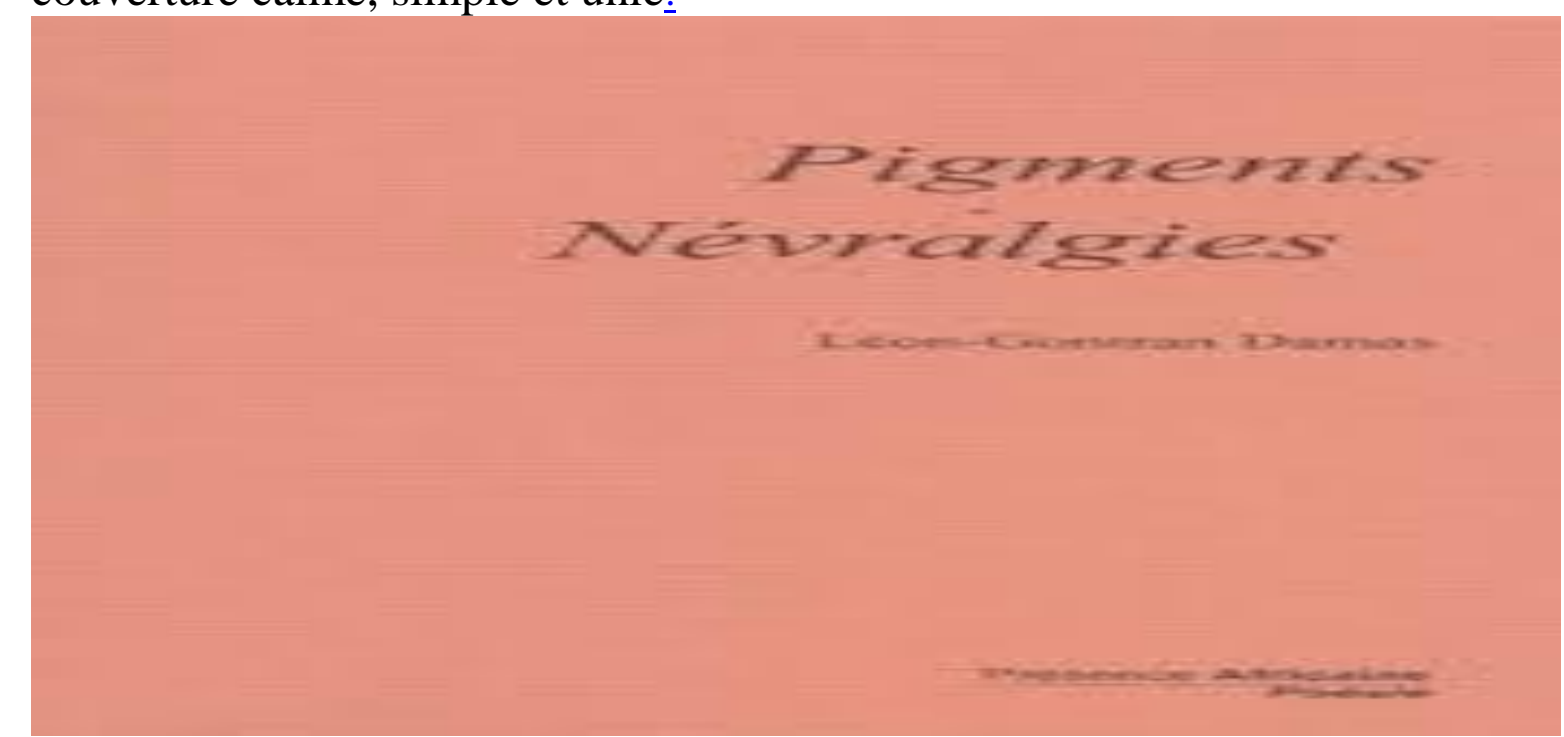

Le choix du rose comme couleur unie de la dernière édition du recueil est emblématique. Le rose s'adapte avec la période finale de la vie du poète et les sujets un peu loin de ce qu'il a suscités dans la période révolutionnaire de sa vie. 
Il s'insère avec "Névralgies" dans la vie sociale des Nègres en gardant son statut comme défenseur de la Négritude.

Cette couleur s'adapte avec l'état d'âme du poète espérant la paix intérieure. Le rose s'avère un signe très important: la paix et la réconciliation du poète avec tous ses anciens ennemis et non la résignation ou l'oubli de ses idéologies. Il souligne sa diaspora et accentue la recherche de son identité en se déplaçant entre plusieurs pays de l'Europe, de l'Afrique et de l'Amérique.

Le choix du rose clair évoque deux problèmes: d'un côté l'état de la réconciliation du poète avec soi-même et avec tout le monde et de l'autre côté une intensité de lumière valorisant la pureté et le murissement de son esprit nègre.

Le rose uni est une couleur qui s'avère de plusieurs signifiés. Elle est la couleur de l'amour, du bonheur, de la délicatesse, de la douceur, de la tendresse et de la féminité. On le lie souvent à l'univers des enfants et à l'évocation de l'entourage enfantin ( $c f$ Zao 2016, 3). Le rose incarne l'état intermédiaire entre les deux états: le Nègre en révolte ou le Nègre en paix.

$\mathrm{Au}$ paratexte vient s'associer un autre élément soulignant de nombreux signifiés de l'idéologie, de la pensée et de l'égo nègre : la typographie.

\section{2- La representative typographique}

Chez Damas, la structure typographique s'avère d'une riche valeur sgnificative. Elle valorise deux figurations de la psychologie abstraite: physique et visuelle. Le blanc et la variété calligrahique des caractères, en italique ou en majuscule, et tout ce qui concerne la mise en page imprimée mettent en évidence une spécificité abstraite et sentimentale chez le poète. Le décodage de cette structure typographique aide à mieux comprendre le message poétique.

Chez les poétes nègres, la typographie accentue" les indices de la subjectivité" (Gardes-Tamine 2001, 4). Tous ces signes typographiques créent un univers animé et expressif soulignant la spécificité du langage de la poésie négro-africaine: "chaque poète ou, plus exactement, chaque ouvre poétique, recourt à ces différentes formes pour construire un univers de signes et de symboles dont la configuration singulière accrô̂t les possibilités sémantiques" (Briolet 1995, 144).

Les poètes négro-africains ont montré la nécessité de trouver un écart stylistique dans la poésie nègre dépendent sur la visualité. Ils ont éprouvé un 
besoin incessant de trouver un moyen implicite et efficace que l'on peut utiliser comme un langage codé. Ils font communiquer aux autres leurs douleurs et valorisent librement leurs coutumes et leurs idéologies. La typographie forme une langue spécifique et un pont entre les Nègres du monde : anglophones et francophones. (cf Kesteloot 2004, 301).

Dans la poésie négro-africaine, la typographie s'avère d'une richesse extraordinaire d'expression et de sensibilité: "à chaque unité typographique correspond une unité sémantique et thématique" (Gardes-Tamine 200133 ). Elle incarne l'image de "l'Afrique debout" (Dieng 2009, 15), c'est-à-dire l'Afrique résistante contre les essais de l'Occident d'effacer sa négresse et son identité par l'imposition de sa culture et de sa civilisation.

Dans la poésie négro-africaine, la typographie représente une "ré-écriture de l'histoire de l'Afrique. Au moyen des lignes courbes du vers et de la strophe, les poètes proposent une historiographie de leur continent à l'aune des aspirations tant individuelles que collectives" (Ngwe, R. (2016). La typographie est la porteuse de la voix d'Afrique, la conservatrice de tout son patrimoine culturel et idéologique. Elle représente une "carte d'identité griotique" ( Delas 2017, 2), c'est-à-dire le narrateur de l'histoire idéologique et culturelle nègre.

Dans "Pigments", la typographie se divise en trois signes essentiels à signifiés très spécifiques. Commençons notre analyse par l'élément le plus expressif et le plus représentatif de sa démarche typographique :

\subsection{Le blanc typographique}

Le blanc est l'élément primordial typographique dans la poésie de Damas. Il représente un langage implicite que le poète emploie pour bien valoriser le drame du nègre opprimé. Sa compréhension garantit l'acquisition de la pensée du poète et de la subjectivité énorme dans la poésie négro-africaine : "l'absence de noir est un signe linguistique à part entier, et même plus que cela dans la mesure où ce blanc-là constitue un signal composite"( Arabyan 1994, 259).

Chez Damas, le blanc exprime toujours des sentiments abstraits. Il donne à la poésie de nouvelles allures poétiques et une émancipation sur le niveau syntaxique et sémantique recompensant celle que le poète espère dans la réalité. Il certifie une situation idéologique et se présente comme témoin sur:

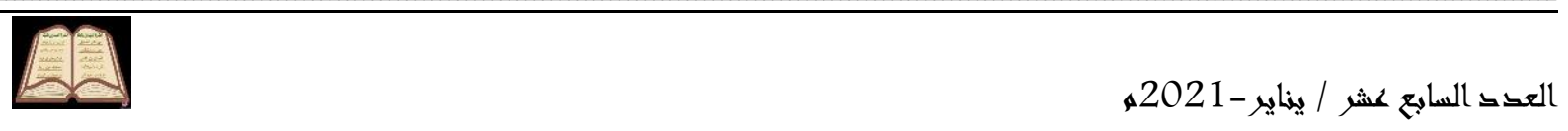




\subsubsection{Le patrimoine oral}

Dans la poésie négro-africaine, les espaces blancs illustrent le patrimoine culturel de leur race, l'aspect folklorique basé sur le chant et sur la danse chez les Nègres en évoquant l'un de plus célèbres instruments utilisés dans le continent africain : le tam-tam.

\section{Ils sont venus ce soir où le}

Tam

\section{Tam}

\section{roulait de}

rythme

en

rythme

la frénésie

(P. Ils sont venus ce soir, 13)

Le «tam-tam », instrument musical emblème du patrimoine oral de toute l'Afrique, est un "tambour de bois africain servant à la transmission des messages ou à l'accompagnement des danses" (www.larousse.fr > francais > tam-tam). La structure des vers imite le rythme du tam-tam. L'inégalité des vers et la différenciation entre le long et le court mettent en valeur une imitation visuelle des vibrations du corps dansant.

L'évocation du tam-tam souligne le désir incessant du retour vers la terre natale. Le tam-tam désigne implicitement la réunion du poète nègre avec son amoureuse, son idole, sa Terre-Mère. Le tam-tam fait un appel à la "reterritorialisation"(Hernandez-Monmarty 2019, 432), c'est-à-dire le retour des Nègres déracinés par les forces odieuses de la colonisation à leur terre natale.

Les mots éloignés progressivement est une imitation visuelle de l'éloignement des sons du tam-tam. Cette imitation donne aux mots un corps matériel décrivant physiquement cet éloignement (cf Hernandez-Monmarty 2019, 232). Il y a aussi une signification implicite de cet éloignement vocalique coïncidant avec l'arrivée des colonisateurs blancs et le commencent de l'effacement d'une culture plus ancienne et plu mûrie.

Le rythme du tam-tam s'adapte avec la démarche militaire des forces de la colonisation et le bruit des pas des soldats. Le tam-tam est témoin des crimes et des massacres que les Blancs ont commis. Il met en évidence l'état pur de l'Afrique avant l'arrivée des colonisateurs et valorise leurs essais d'effacer

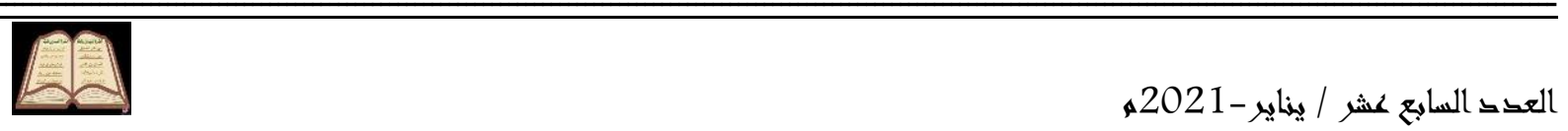


l'identité culturelle de l'Afrique. Le poème est dédié au poète nègre, Senghor après son accord sur l'envoi des Combattants Sénégalais pour soutenir la France contre le Nazi. Le même rythme se manifeste clairement dans les vers suivants:
Aux Anciens Combattants Sénégalais
aux Futurs Combattants Sénégalais
à tout ce que le Sénégal peut accoucher
de combattants sénégalais futurs anciens
de quoi-je-me-mêle futurs anciens
de mercenaires futurs anciens
de pensionnés
de galonnés
de décorés
de décavés
de grands blessés
de mutilés
de calcinés
de gangrenés
de gueules cassées
de bras coupés
d'intoxiqués
et patati et patata
et cætera futurs anciens (P. Et cætera, 79))

La disposition du blanc et l'écriture, quelquefois, d'un seul mot sur la ligne figurent un rythme soudainement coupé concrétisant visuellement et audiblement la marche militaire réglée des soldats dans leur avancement. L'expression "onomatopéique "patati et patata" désignant leur bruit et leur bavardage met en évidence cet avancement. Le couple contradictoire "anciens-futurs" que le poète emploie pour faire rappeler aux combattants leur passé en luttant contre la France coloniale. Cette situation s'est transformée en combattant en faveur de leur oppresseur.

L'accumulation successive des lignes courtes souligne les sacrifices de ces soldats dans leurs combats en faveur de la France, ils sont des "blessés-mutilés". Damas évoque aussi les anciens sacrifices de combats de libération contre la France qui demande l'aide de ceux qu'elle a opprimés. Le blanc valorise une 
"fonction dramatique" (Hernandez-Monmarty 2019, 33) en mêlant les sonorités du tam-tam avec le bruit des guerriers et leur mouvement.

Ce ryhme vertical ressemble au martèlement violent et évocateur qui matraque les colonisateurs. Il évoque une longue liste de leurs crimes : violer, voler, souiller, opprimer, tuer...etc. Cet enjambement à la ligne dresse une liste honteuse et scandaleuse des crimes des Blancs : sadisme, malpropréité, saleté. Le blanc tyographique encadre les génocides des Blancs colonisateurs et représente un enjambement d'un crime à l'autre. Cette répartition du blanc présente le poème comme une colonne mettant en évidence une verticalité frappante qui encadre le texte entre deux signifiés : la lourdeur et la lenteur.

Le patrimoine oral s'accomplit avec le rythme jazzique dans ces vers :

Et mon visage brille aux horreurs du passé

et mon rire effroyable est fait pour repousser le spectre des

et ma voix qui pour eux chante

est douce à ravir

l'âme triste

de leur por-

no-

gra-

phie (P. Shine, 66)

Ce poème est dédié à Louis Armstrong, inventeur du rythme jazzique. Or le jazz est à la fois un mouvement sonore et corporel. Il se caractérise par sa liberté et exprime implicitement plusieurs signifiés concernant le monde nègre: la douleur, l'oppression, plainte, ironie, révolte et revendication (cf Boross, www.offjazz.com).

Sur le ton du rythme jazzique caractérisé par la succession alternative des pieds des danseurs, Damas souligne le passé scandaleux des Blancs: " Les vers de Damas épousent le rythme syncopé du jazz et les ressassements du blues, autant qu'ils égrènent les désirs et rêves inassouvis d'un poète, métis, exilé, amoureux, à la fois solitaire et solidaire" (Saïd 2016, 4)

Le poète compare les Blancs aux lévriers, aux chiens qui s'en vont à la poursuite des nègres fugitifs. Le couple "marronnage-lévrier" désigne ce signifié dramatique. Le poète accentue le passé odieux de la colonisation par le chant, la danse et le rire. Il s'attache aux origines nègres et proclame sa dénonciation de la 
civilisation du monde blanc. Le signifiant"pornographie" coupé sur quatre lignes souligne, sur le souffle du jazz, l'immoralité, la grossièreté, la licence sexuelle et l'impudicité de la civilisation occidentale.

Cette manière d'écriture, qui ressemble aux poèmes de Calligrammes d'Apollinaire, met en valeur le lien entre la forme physique et l'expérience corporelle et sensuelle du poète (cf Hernandez-Monmarty 2019, 61). Le cas est le même dans les vers suivants:

Et veille mon cœur

de mon rève qui se nourrit du bruit de leur

dé-

$$
\text { gé- }
$$

né-

rescence

est plus fort que leurs gourdins d'immondices

brandis ( P. Shine, 66)

Dans l'idéologie nègre, la musique du jazz est toujours le miroir de ses maux. La disposition des mots s'adapte avec le mouvement du corps du poète, pieds et mains, avec le glissement du danseur qui joue sur l'espace blanc de la page. Le mot est divisé d'après le souffle du jazz (cf Hernandez-Monmarty 2019, 222-223). Le poète met en valeur la "dégénérescence" des Blancs encadrée par le rythme frappant du jazz et la successivité du mouvement des pas. Ce rythme désigne un tableau magnifique où s'alternent l'oppression et l'injustice pour les Nègres, et la corruption et la décadence des Blancs.

Son refus de s'assimiler aux Blancs s'est accentué par le même rythme jazzique :
J'ai l'impression d'être ridicule
dans leurs souliers
dans leurs smoking
dans leur plastron
dans leur faux-col
dans leur monocle
dans leur melon

(P.Solde, 41)

Ce poème est une forte dénonciation de la loi de l'assimilation imposée aux Nègres pour suivre les manières de vivre des Blancs. Le blanc typographique 
valorise le rythme vertical martélant sur le même signifié. Il traduit le refus de Damas à l'assimilation aux Blancs. Il refuse tous les aspects de leur civilisation: leurs vêtements, leurs maisons, leurs meubles, leurs besoins, leurs instincts, leurs manières de vivre et même leurs sentiments. Ce signifié s'avère clairement dans les vers suivants:

\title{
Blanchi
}

\author{
Se peut-il donc qu'ils osent \\ me traiter de blanchi \\ alors que tout en moi \\ aspire à n'être que nègre \\ autant que mon Afrique \\ qu'ils considèrent camriolée
}

\section{Blanchi ( P. Blanchi, 59)}

Le blanc met en valeur le participe qui traduit un horizon d'attente suspendu entre le présent et l'avenir, un état qui ne donne pas une limitation temporelle. Le participe déduit plus vite et plus loin l'intimité psychologique pure (cf Décaudin 2013, 193). Le retour du participe "Blanchi" qui est une reprise du titre du poème et qui s'emploie comme refrain entre les différentes srophes représente un retour périodique et régulier de l'idée essentielle dans le poème.

Ce retour du participe "Blanchi" met en valeur le tourment du poète, obligé de vivre par la loi comme les Blancs. Le blanc typographique valorise une idée d'infériorité et de disqualification: le nègre ne peut continuer sa vie que par l'abandon de son identité et par l'assimilation aux Blancs. Le refrain se revêt ainsi d'une valeur rythmique qui donne au poème un martèlement régulier comme un "aliment nécessaire de sa mémoire" (Amon et Bomati 1994, 126) sur une impression, sur une pensée ou sur un jugement. Le poète refuse de vivre dans un masque blanc. L'expression "mon Afrique" renforce ce signifié et illustre la confirmation du poète sur ses origines nègres. Ce signifié se traduit clairement dans les vers suivants:

"les mains effroyablement rouges

du sang de leur ci-vi-li-sa-tion" " P.Solde, 42) 
La calligraphie du signifiant "civilisation" avec des syllables séparées par un trait d'union impose une cassure à l'intérieur du mot (cf Lapierre 2016, 64). Le poète nègre met en relief les causes de son refus de l'assimilisation aux Blancs et à leur civilisation qui est souillée du sang des Nègres. Ce trait d'union impose une lecture consciente de chaque syllable qui devient une reprise du signifié original et une cristallisation de la problématique des Nègres. Le même signifié s'affirme clairement dans les vers suivants où la disposition syllabique du signifiant sur les lignes permet une lecture lente et dense du terme:

\section{où cacher mon visage}

ma honte

de

la

Ré

$\mathbf{a}$

li

té (P. Réalité, 71)

La cesure rythmique coupe les segments sans détruire le signifié logique du contexte (cf Lapierre 2016, 62-63). Les mots syllabiquement dispersés doivent être intégrés et retenus consciemment. La répartition de la "réalité" souligne la cruauté et le fauvisme envers les nègres et encadre leurs douleurs.

Le blanc typographique figure comme une force représentative de la pensée et de l'intimité nègre, une intimité psychologique morcelée et disperséé par la réalité cruelle. Il évoque un contenu morcelé et une communication déchirée avec le monde : "relation d'intermettence entretenue par le poète avec le monde" (CASTIN 1998, 153). Le poète, à travers la décomposition et le déchirement des mots, dresse implicitement une invitation aux lecteurs de partager ses maux et ses douleurs.

La successivité des lignes courtes composées d'un seul mot isolé dresse au lecteur un choc résultant de l'alternance des informations qui oblige le lecteur de suivre un certain itinéraire de lecture:

\section{ils ont cambriolé l'espace qui était le mien la coutume \\ les jours \\ la vie}

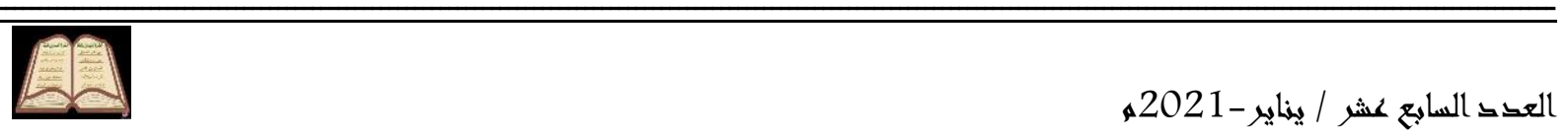




\section{la chanson \\ le rythme \\ l'effort \\ le sentier \\ l'eau \\ la case \\ la terre enfumée grise \\ la sagesse \\ les mots \\ les palabres \\ les vieux \\ la cadence \\ les mains \\ la mesure \\ les mains \\ les piétinements}

les sols (P.Limbé, 44)

Ce rythme vertical accumule les maux du poète qui souffre de l'enlèvement de sa patrie et de tout ce qui représente son identité. La musique du jazz rythmisant la danse, le chant et le cri accentue le mal du poète et fait ressentir un énorme tourment psychologique. La cassure des lignes, composées parfois d'un seul terme, coïncide avec les courtes sonorités rapides et valorise chez le poète une "poétique corporelle" (Hernandez-Monmarty 263) mettant en évidence une harmonie entre la pensée et le mouvement du corps. Cette danse folklorique sur le ton du jazz représente pour le Nègre une sorte de rédemption de tous ses maux. Ce signifié s'avère bien clair dans les vers suivants qui cristallisent le cri et la douleur du nègre tourmenté:

\section{ils me l'ont rendue}

la vie

Plus lourde et lasse ( $P$. La complainte du nègre, 47$)$

La disposition du blanc est une traduction d'une anxiété ou d'une tension émotionnelle. L'alternativité des vers inégaux valorise un souffle rapide figurant la colère ou une psychologie émotionnelle. Ce rythme accéléré, ressemble aux cascades successives exprimant les mouvements de la pensée et du cœur. Le détachement du complément "la vie" évoque les maux que le Nègre endure alors 
que le détachement du groupe adjectival "Plus lourde et lasse" encadre la qualité de ces maux et de ce tourment.

Le blanc et le rythme se communiquent. L'armature rythmique émane d'une intimité psychologique d'angoisse et de d'étouffement. Le ralentissement causé par la transition d'un vers long à un autre plus court produit un "écartement analytique" (Grammont 1965, 75), c'est- à-dire un frappement tenté sur quelques signifiés : "tristes", "mémoire" et " vie": "le seul guide pour le poète est le rythme,non pas un rythme appris, gratté par mille règles que d'autres inventèrent, mais un rythme personnel qu'il doit trouver en lui-même" (Dessons 1991, 117):

\section{De n'avoir jusqu'ici fait détruit bati \\ osé \\ à la manière \\ du juif \\ du Jaune \\ pour l'évasion organisée en masse de l'infériorité (P. Réalité, 71)}

La répartition du blanc est une valorisation du regard d'infériorité des Européens vers les autres races. La verticalité du poème est une ponctuation visuelle de chaque race opprimée, le poème devient une colonne regroupant tous les damnés de la terre: Juifs et Jaunes, désignant la race asiatique.

Le détachement et l'isolement du mot "infériorité" souligne l'amertume qu'éprouve le poète. Mais ce dernier désigne à travers la disposition du blanc le remède de tous les problèmes des Hommes : la danse qui se place "parmi les signes qui permettent aux hommes de se communiquer entre eux ce qu'ils éprouvent, certains concernent la vue, la plupart l'oüe, et un tout petit nombre les autres sens." ( Acone 2017, 103).

Mes amis j'ai valsé

valsé toute mon enfance

Danube blanc

Danube rouge

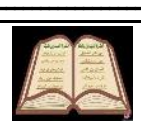




\section{Danube rose \\ Danube blanc \\ rouge \\ vert \\ rose \\ au choix (P. Nuit blanche, 57)}

Le blanc et la reprise anaphorique de l'expression " Danube" colorisé de toutes les couleurs valorisent une danse sur le ryhme syncopé, court et rapide du jazz. Cette danse unit tous : "les âmes, damnées ou pénitentes, se tordent de douleur, pleurent et incarnent par leurs corps agités, dansants, la douleur et la souffrance" (Acone 2017, 102).

D'après Damas, la danse s'adapte avec l'intimité tourmentée de l'âme, et le débarrasse de ses péchés. Ce rythme sur le ton de la danse de la valse et du jazz:"incarne les passions et son cheminement intérieur par l'évocation de mouvements corporels, de mimiques et de danse" (Acone 2017, 102).

Dans la croyance nègre, la danse et le chant sont un moyen de remporter la faveur des Hommes et de Dieu. Danser, c'est une sorte de résistance contre les ennemis pour les surmonter. Les battements du tam-tam, symbole de l'identité et de la culture noire originale et du jazz, slogan de l'union de tous les nègres du monde, encadrent une graphie déchirée ou un rythme cassé sur la page s'avèrant d'un "dérèglement psychique" (Toh Bi 2016,3).

La danse valorise un rituel religieux et un sentiment céleste s'avérant très clair avec l'évocation du temps de l'enfance:

\subsubsection{Le souvenir d'enfance}

Le blanc met en valeur des pauses temporelles dans la vie du poète qui sont spécialement évocatrices du temps de l'enfance. L'enfance représente pour le poète un univers d'innocence, de pureté dont il est le roi. L'évocation de l'enfance met en évidence un désir frappant de retour aux origines et un refus irrévisible du déracinement. Le bonheur est toujours lié à l'évocation de l'enfance:

\section{Rendez-les-moi mes poupées noires} mes poupées noires poupées noires noires

noires (P. Limbé, 45)

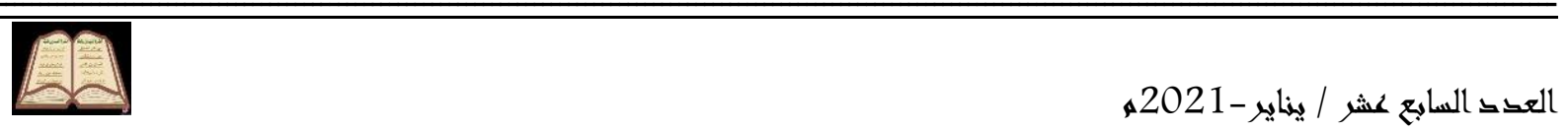


L'évocation des souvenirs de l'enfance est toujours liée à l'évocation de la terre natale et du temps de l'origine qui est toujours remarquablement présente chez Damas : "l'image d'une enfance volée se superpose à celle de l'Afrique cambriolée. Le drame personnel rejoint le drame historique accusant le rapt originel, la castration, le démembrement d'un corps individuel et collectif" (Saïd 2016, 4).

La disposition du blanc avec la répétition frappante à la fin de chaque vers de "noires" met l'accent sur l'identité nègre qui devient un leitmotiv dans la poésie de Damas. Il accuse l'Occident de violer son enfance caractérisée par la poupée noire "Limbé" que les Blancs achètent pour leurs enfants ( cf Moudileno 1997, 182). L'image de cette poupée déracinée de sa terre natale ressemble aux Nègres qui ont eu le même destin. Cette poupée est une reprise et une activation de la mémoire collective de tous les enfants noirs.

La plupart des poètes nègro-africains donnent une importance majeure au thème de "la nostalgie pour le royaume de l'enfance" (etudianteeternelle.wordpress.com > leon-damas, 2). À l'inverse, Damas, poète révolutionnaire dans son esprit et dans son écriture, évoque son enfance avec amertume et avec colère. Son enfance lui fait évoquer son passé mélancolique. La succession des souvenirs s'adapte avec la disposition du blanc sur la page:

Ma mère voulant d'un fils très bonnes manières à table

Les mains sur la table

le pain ne se coupe pas

le pain se rompt

le pain ne se gaspille pas

le pain de Dieu

le pain de la sueur du front de votre Père

le pain du pain

au vu de tout le monde

et puis tenez-vous droit 


\section{un nez bien élevé ne balaye pas l'assiette ( P.Hoquet, 35-36)}

Le blanc valorise la figure maternelle mettant et évoque l'ennui de l'enfant des tentatives de sa mère de lui imposer toutes les habitudes des enfants blancs en croyant qu'elle le rend plus raffiné et plus civilisé. On doit avoir les bonnes mœurs de table: ne pas couper le pain, ne pas finir complètement le plat, ne pas faire de bruit avec les fourchettes contre les dents, ne pas baisser le nez mais le garder bien élevé, s'asseoir tout droit à table en mettant les mains sur la table. La successivité verticale des vers dresse la liste des instructions de la mère pour faire de son enfant une figure pareille aux Blancs. Les vers déchirés, successifs et très courts sont annonciateurs du refus de l'enfant des règles imposées de sa mère.

La répétition anaphorique du signifiant "pain", aliment de base de la table et de la culture française, souligne la tentative fidèle de cette famille de vivre à la française. L'existence du pain représente dans la mentalité occidentale un rituel religieux connotant le corps du Christ et une reprise de sa parole: "Je suis le pain vivant, si quelqu'un mange de ce pain, il vivra éternellement" (Cabanis 2018,1).

Les ordres de la mère représentent pour Damas une infériorité raciale. La mère les impose comme un contrat social de vivre dans la maison et elle blâme son enfant qui refuse de les exécuter. Ce signifié s'est bien manifesté dans les vers suivant:

\section{Ma mère voulant d'un fils}

fils de sa mère ( P.Hoquet, 37)

La successivité des vers coupés et dentelés valorise le ton ironique des instructions maternelles que l'on considère comme de bonnes manières de vivre. Le poète utilise le blanc typographique pour encadrer une ridicule et une exaspération de quelques instructions maternelles ( cf Kesteloot 2004, 115):

- Confectionner les lecons de la musique et spécialement du violon:

Ma mère voulant d'un fils très do

très ré

très mi

très fa 


\section{très sol \\ très la \\ très si \\ très do \\ ré-mi-fa \\ sol-la-si \\ do \\ Il m'est revenu que vous n'étiez encore pas \\ à votre leçon de vi-o-lon \\ Un banjo \\ vous saurez qu'on ne souffre chez nous \\ ni ban \\ ni jo \\ ni gui \\ ni tare}

Le blanc valorise la voix de la mère qui sépare entre deux catégories de la musique : la musique classique européenne concrétisée par le violon et le solfège s'appuyant sur l'apprentissage de la musique, de la perfection du système de notation musicale, du chant et des instruments musicaux. La deuxième catégorie s'avère avec la musique qui inclut l'entourage nègre comme la guitare et le banjo, instrument originaire de l'ouest de l'Afrique venu avec les esclaves nègres.

L'enfant ne peut pas utiliser sans peur de reproche et de colère ses jeux bruyants comme le banjo et la guitare. Il est obligé d'utiliser le violon. On l'insère obligatoirement à la religion, à l'histoire et à la musique. Ce contraste qu'aborde le poème met en évidence l'angoisse du poète et de son enfance déchirée entre deux mondes : il n'éprouve complètement ni son identité nègre ni l'identité blanche qui lui est imposée.

Le déchirement et le morcelement des mots sur les lignes soulignent une distance blanche entre les parties du discours entre la mère et son enfant. La décomposition syllabique visualise derrière elle une caricature de quelques items 
de la civilisation européenne agressive. Le morcèlement graphique met en évidence une "peudo-culture", une autre idéologie plus forte et plus solide, c'est le Nègre fier de son identité (cf etudianteeternelle.wordpress.com > leondamas, 4).

- Respecter les règles de la religion catholique:

Et puis et puis

et puis au nom du Père

du Fils

du Saint-Esprit

à la fin de chaque repas (P.Hoquet, 36)

Le blanc typographique valorise une coincïdence entre le langage et la spiritualité religieuse. Il met en évidence un "rituel de la table" (Donada, croire.la-croix.com > Vivre-en-chretien, 1) tiré des traditions catholiques qui dominent la société française fortement attachée au christianisme. Le blanc encadre l'effort de la mère pour habituer son fils nègre à réciter, dans les repas, la prière d'action de grâce caractérisant la société des Blancs. Le même signifié se manifeste dans:

\section{Ma mère voulant d'un fils mémorandum}

\section{Si votre leçon d'histoire n'est pas sue vous n'irez pas à la messe dimanche avec vos effets des dimanches (P.Hoquet, 36)}

L'isolation du mot dimanche encadre l'attachement au christianisme. La participation à la messe du dimanche est un des signes de l'Homme blanc et de son sublime (cf Buata 2008, 178). Ce signifié est bien évident avec le signifiant "mémorandum" qui est encadré par un vaste espace du blanc qui le suit. La participation à la messe est une des bases de la culture française blanche. Damas ridiculise le desir de sa mère de participer à la messe qui ne s'émet pas d'une vraie croyance, mais d'une volonté de l'assimilation et c'est le nœud de la souffrance du poète.

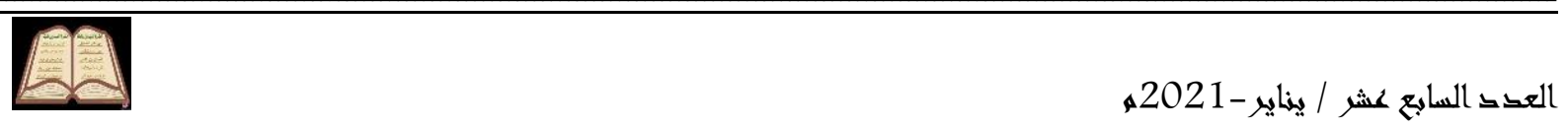


- Refus de l'éducation imposée, de la prédilection et la supériorité de la civilisation francaise

\section{Taisez-vous \\ Vous ai-je ou non dit qu'il vous fallait parler \\ français \\ le français de France \\ le français du Français \\ le français français (P.Hoquet, 37)}

Le blanc typographique met en valeur le regret du poète sur son enfance opprimée et déracinée de sa culture native. Il est obligé de recevoir l'éducation des Français et de parler à leurs manières. Les colonisateurs essaient d'effacer l'identité nègre. Ils imposent le français afin d'insérer les Nègres dans leur civilisation.

Le blanc typographique met en relief cette enfance désastreuse du poète déchirée entre deux identités en conflit. Il souligne un mélange d'humour, de satire, de colère et d'exaspération. Ce signifié est bien évident avec la répétition du signifiant "désastre" évoquant le mal psychologique du déchirement de l'enfant entre deux identités.

La souffrance et la douleur du poète s'accomplissent, à travers le blanc typographique, par la cristallisation de ses désirs de retour vers la terre natale, berceau du paradis de son enfance :

\subsubsection{La recherche de l'identité nègre}

Le blanc typographique met en valeur l'image du Nègre déraciné et perdu cherchant son identité et son appartenance à la terre natale. Le blanc met en valeur sa quête:

\section{hier}

\section{sans complexité}

\section{hier}

quand est venu l'heure du déracinement (P. Limbé, 45)

La disposition du blanc représente un encadrement d'un moment triste dans la vie du poète, le moment de sa déterritorialisation et de son déracinement de son pays natal. L'écriure de "hier" sur la ligne au début est une évocation du passé heureux de l'enfance et de sa patrie où

$$
\text { العكَ السابع عشر / يناير - 2021ه. }
$$


dominent le bonheur et la liberté. Sa reprise valorise un principe fixe chez le poète: rien ne peut récompenser son désir de retour à son paradis:

\section{Sur l'océan}

je me suis réveillé

nuit noire

épris

sans jamais rien saisir

de tout ce que racontait le vent sur l'océan

nuit noire

\section{Pendant qu'un bateau foule l'écume}

et va

\section{va son detin de roulure} sur l'océan

\section{nuit noire (P. Le vent, 29)}

Le blanc traduit le sentiment de la perte qu'éprouve le poète déraciné de son paradis natal. Il évoque le moment de sa déportation de sa terre vers la France. La répartition du blanc et l'écriture de l'expression "nuit noire" traduisent le labyrinthe et la perte qu'éprouve le poète depuis cette nuit qui est une figuration du temps le plus odieux et le plus mélancolique de sa vie. Le déictique " hier" renvoie à un double signifié concernant soit son enfance heureuse soit la date noire de son déracinement. Le blanc met en évidence le voyage qui insère le poète dans un état de malaise, de solitude et de perdition. L'expression " sur l'océan" met en relief le sentiment de perte du poète après son déracinement.

La représentativité typographique s'avère d'une grande importance pour renvoyer au statut du nègre en recourant à l'usage de la majuscule:

\subsection{La majuscule}

D'habitude, la majuscule est mise au début de la phrase. Mais dans la poésie négro-africaine, on observe son emploi fréquent à l'intérieur de la phrase. L'écriture poétique de Damas se distingue par l'usage exagéré de la majuscule. Il l'emploie comme un des outils de mise en évidence en déviant avec l'ensemble 
calligraphique pour attirer l'attention du lecteur avec un grand "délassement de la vue" (Catach 1994, 83).

Chez Damas, la majuscule peut encadrer une contestation, un refus ou une révolte. Elle impose sur le lecteur un arrêt obligatoire devant certain changement graphique soit en caractère soit en mesure. Il doit s'arrêter pour recevoir soigneusement ce changement de caractères. Ce changement calligraphique peut évoquer un signifié très important. Sa visibilité est condensée avec l'abscence totale de la ponctuation. Elle sert comme repères dans ce monde chaotique du poème:

"des yeux
la frénésie des mains
des pieds de statues

DEPUIS

combien de MOI MOI MOI

sont morts

depuis qu'ils sont venus ce soir (P.Ils sont venus ce soir, 13)

Cette reprise du signifiant "moi" en gros caractères et en majuscule valorise l'histoire odieuse et sanguine que les Blancs ont tracée dans la mentalité des Nègres. Cette répétition avec la majuscule impose une forte contestation sur les massacres commis par les colonisateurs et met en évidence le contrat de fraternité unissant tous les Nègres et cristallise la cruauté des colonisateurs.

La répétition du pronom"moi" manifeste d'une manière aiguë la multiplication et la successivité interrompue de la tuerie et de l'oppression (cf Lapierre 2016, 65).

La répétition du déictique accentué du "je", "moi", est une manière d'amplifier non le ""je" du poète mais d'amplifier les morts qui ne sont en fin de compte qu'une image du "moi". Aussi met-elle en relief les nombreuses générations qui ont été enfouies et oubliées dans les longues années de résistance contre les oppresseurs blancs.

L'écriture de l'averbe de temps "depuis" tout en caractères gras avec une calligraphie épaisse au milieu du poème met en évidence les massacres et les génocides commis par les colonisateurs. L'écriture d'une manière visuellement différente à l'intérieure d'une structure calligraphique cohérente s'adapte avec la perturbation de la vie des africains et avec le déchirement qu'éprouvent les Nègres dès l'arrivée des colonisateurs blancs. 
L'adverbe "Depuis" rend le rôle d'une limite séparatrice entre deux périodes : le début d'une ère d'oppression et de sang et la fin d'une autre pleine de bonheur, de chant et de danse sur les sonorités du tam-tam ( cf Toh Bi 2016, 2). Ce signifié se manifeste clairement avec le déictique "Hier". Dans un contexte différent, le poète souhaite revivre le bonheur et le temps passé de l'enfance. Il évoque les souvenirs de sa terre natale et il souhaite capter le "Hier" plein de bonheur:

\author{
"redevenu moi-même \\ nouveau moi-même \\ de ce que Hier j'étais (P. Limbé, 44)
}

L'écoulement du sang et l'égorgement des Nègres soit par leur fonctionnement comme des esclaves chez les Blancs ou par leur exploitation dans la plantation des fermes et dans la fabrique des usines se montrent clairs avec la répétition du déictique accentué "moi"(Kesteloot 2012, 45).

La majuscule encadre aussi une mémoire odieuse chez le poète et dresse un cri piquant de douleur. La calligraphie de l'usine et sa distinction avec une majuscule accentue cette mémoire douloureuse:

"Et flûte

Flûte de roseau

jouant sur les mornes des airs d'esclaves

pendant qu'aux savanes

des bœufs sagement ruminent

pendant qu'autour

des zombies rôdent

pendant qu'ils éjaculent

les patrons d'Usine

pendant que le bon nègre

allonge sur son grabat dix à quinze heures d'Usine"(P.Rappel, 63)

L'usine tient une mémoire malheureuse chez le poète. Elle évoque l'image de l'exploitation des Blancs pour les Nègres. Elle traduit un tableau misérable de la vie du Nègre humilié et extrêmemt exploité, de l'usage esclavagiste des Nègres et leur exploitation corporelle. Ils doivent déployer des efforts insupportables. Ils se transforment en vue de la fatigue du travail à des " 
zombies", c'est- à-dire des morts vivants, des cadavres errants. Dans la conscience collective des Nègres, l'unsine est l'aspect fondamental de la corvée, de la cruauté de la colonisation et de l'épuisement des trésors du Continent Noir.

Dans la poésie de Damas, la masjuscule peut se revêtir aussi d'une valeur allégorique. Dans la poesie nègro-africaine, la figure maternelle représente une allégorie de L'Afrique. Elle met en évidence la mémoire éternelle du paradis de l'enfance et le sein de la terre natale.

\section{" Ma Mère voulait d'un fils très do très mi très fa très sol} ............. (P. Hoquet, 38)

Chez Damas, la Mère est l'équivalent de 1Afrique, elle devient son "archétype" et le symbole de la vie pour les Nègres. Chez lui, la mère avec sa sévérité douce représente la loi absolue organisatrice du comportement et de la vie des Nègres. Elle est la protectrice de la race. Elle est l'accouchée des Nègres et la conservatrice de leurs générations successives. Elle occupe sacrement une place céleste dans la mentalité collective des Nègres. Elle est glorifiée comme source de la poétisation de la plupart des poètes négro-africains. Elle représente la base de l'idéalisation et de la beauté féminine éternelle (cf Boyce-Davies et Ogundipe 1995, 137).

Chez Damas, la majuscule met en relief le but essentiel de sa poétique, le conflit des peaux et des pigments, autrement dit la discrimination raciale:

Passe pour le crétin d'Allemand de se promettre d'avoir la peau du Français

de se promettre d'avoir la peau de l'Allemand et d'en faire des sauts de lit ( $P$. Sur une carte postale,77)

" De n'avoir jusqu'ici rien fait détruit

à la manière

du Juif du Jaune 


\section{pour l'évasion organisée en masse \\ de l'infériorité (P. Réalité, 71)}

La majuscule met en évidence ici l'oppression raciale existée dans le monde soit pour le juif soit pour le Jaune, citoyen de l'Asie. Le poète se moque de la haine et de la folie qui existent même entre les Européens "FrançaisAllemands" malgré qu'ils ont la même couleur de la peau. Parmi ce chaos racial, il met en évidence sa fierté de son statut nègre. Ce signifié, qui exprime bien l'essence du mouvement de la Négritude, s'avère bien clair dans les vers suivants:

\section{" alors que tout en moi}

aspire à n'être que nègre ( $P$. Blanchi, 59)

Dans Pigments, on renconre un phénomène général, c'est l'écriture de tous les caractères des titres en majuscule: LE VENT, REALITE, SOLDE, CAPTATION, COMPLAINTE DU NEGRE...etc. Ce titre, suivi souvent d'un espace blanc est repris dans beaucoup de poèmes comme refrain pour signaler une certaine valeur sémantique.

Cette technique présente le titre comme résumé de tout le poème, une conséquence retenue de l'avant lecture et aborde implicitement une orientation du lecteur pour prendre conscience de quelques pensées (cf Lapierre 2016, 65).

En ce cas, le titre représente souvent la phrase porteuse de toute la pensée du poète mettant en valeur un contexte historique, un lieu, un personnage. Il inscrit d'avance avec le lecteur un contrat de lecture indiquant un code quel'on doit utiliser pour pouvoir poursuivre ou comprendre le message du poète. $\mathrm{Ce}$ signifié se montre explicitement dans le vers suivant:

" de tout ce qui m'émmerde en gros caractère" ( $P$. Pour sûr, 53)

La diversité des aspects typographiques peut souligner des signifiés spécifiques concernant la conception nègre qui s'avère bien claire avec l'usage rare de l'italique:

\section{$2.2 \quad$ L'italique}

Dans Pigments, l'italique est rarement utilisé. Il produit une influence semblable à la majuscule. Le poète s'en sert pour montrer une différence, un changement, une mise en évidence, un sous-entendu, une distinction d'une telle idée. Chez Damas, l'italique s'emploie pour concrétiser un changement rythmique en isolant quelques mots valorisant certains signifiés: "Sa fonction est celle d'un soulignement linguistique, c'est-à-dire que sa valeur est tout à la fois rythmique et sémantique "(DESSONS 1991, 142):

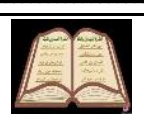




\section{Non monsieur}

vous saurez qu'on ne souffre chez nous

\section{les "mulâtres" ne font pas ça \\ laissez donc ça aux "nègres" (P. Hoquet, 38)}

" Mulâtre", signifiant le statut du nègre dont l'un des parents est blanc ou dont les deux sont "mulâtres"(cf Chaulet-Achour 2012, 195). L'écriture en italique des "mulâtre et nègre" met en évidence le déchirmement entre deux identités dont a souffert le poète durant sa vie: être Nègre et être l'image du Blanc. L'italique, par sa différenciation calligraphique, rend visuellement le conflit entre deux identités.

En recourant à la majuscule ou à l'italique, Damas éblouit le lecteur par des variations graphiques, dotées d'une valeur à la fois rythmique et sémantique. Ils produisent une rupture avec le contexte calligraphique commun pour mettre en valeur des signifiés importants. Ils accentuent ce changement typographique mettant en relief la pleine valeur du signifiant. Cette armature typographique "ouvre l'architecture textuelle de la poésie moderne à la présence d'une concrétude, aux enchevêtrements vécus d'une expérience, bref, qui ancre la page dans l'existence, dans le rapport, infiniment varié, d'un sujet à un monde" (Briolet 1995, 152).

La diversité typographique dans Pigments accentue une contestation et un refus dans l'intimité du poète de la réalité vécue qui se reflète sur la forme du poème: "une description originellement conçue comme transcription d'un espace imaginaire dans un espace textuel" (Briolet 1995, 152).

\section{Conclusion}

Comme le destin de chaque novateur, talentueux et en même temps révolté, Damas sent un besoin incessant et martélant de vivre différemment dans la société. Ce besoin le pousse à travailler toujours sur un système devié de tout ce qui est commun dans son entourage spirituel ou poétique, le motive extrêmement d'écrire différemment de ses contemporains.

Les poèmes de "Pigments" sont un panorama historique valorisant les relations de conflit et d'incommunicabilté entre les Nègres et les Blancs. Damas inscrit cette longue histoire à travers le paratexte et la typographie qui se présentent comme un moyen efficace de communication. Ils représentent une reproduction de l'identité nègre et un reflet de l'intimité psychique.

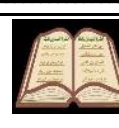


A travers des décennies des années dans lesquelles, le Nègre est accusé de la négativité et du silence en raison du manque de culture et d'éduction. Seul l'Homme blanc, civilisé et bien cultivé, est le roi de la langue et de l'expression. Damas a détruit cette croyance en transformant sa poésie en un champ de challenge raciste entre les Blancs et les Nègres pour prouver l'excellence et la supériorité. Il prouve qu'à travers le silence et la négativité présupposées, il va inventer un langage extrêmement expressif, un méta-langage que les Blancs, eux-mêmes, vont déployer des efforts pour pouvoir l'atteindre. D'après lui, la langue classique représente un carcan tyrannique en étouffant la liberté de l'imagination et de la création.

Le paratexte et la typographie deviennent l'outil de la poésie négroafricaine pour rendre la supériorité de la parole et de l'expression aux Nègres accusés d'être muets. Ils deviennent leur porte-parole et leur choix de résistance puissante contre la tyrannie de L'Homme blanc.

Chez Damas, le choix du titre est bien représentatif et bien distinctinctif en réalisant les trois conditions d'après la pensée de Gérard Genette. Il identifie en premier lieu la nature de l'ouvrage et l'idéologie de l'auteur. Puis, il dévoile le contenu de l'ouvrage. Il est bien opératif de son contenu. En bref, désignation, valorisation du contenu et séduction des lecteurs.

Les éléments paratextuels jouissent de propres caractéristiques encadrant une base idéologique principale : la Négritude. Ils mettent en valeur la conception de la fierté du statut nègre, le déchirment et le morcèlement qu'éprouve le Nègre après son déracinement de sa terre natale. Tous ces éléments accentuent et ponctuent le poème d'informations redondantes guidant le lecteur et formant un contrat de lecture permettant de recevoir le signifié nègre. Ils lui présentent une anticipation interprétative du contenu du poème.

Le changement typographique souligne un refus révolutionnaire contre tout ce qui assujettie l'homme: le racisme odieux, l'idéologie politique bâtie sur le pouvoir absolu dont le fruit était l'apparition de plusieus régimes oppresseurs. La typographie met en évidence une révolte contre la situation immorale et la réalité injuste imposée par la colonisation. Comme tentative de changer cette actualité, la variété typographique concrétise un cri de révolte contre cette réalité imposée. Pour Damas, la révolte contre le système classique de l'écriture est une concrétisation de son refus du système mondial produisant des forces fascistes et 
tyranniques. Pour Damas, l'écriture poétique est le reflet de son personnage comme Nègre révolté.

Dans "Pigments", le paratexte et la typographie mettent en évidence la manière dont le poète s'en sert pour combler le déchirement qui a laissé ses empreintes dans la vie d'un nègre errant: soumission, colonisation, assimilation, aliénation, discrimination raciale, oppression et effacemenet de la culture noire. Ils mettent en valeur le conflit intérieur piquant chez le poète qui le pousse à changer sa réalité.

Le blanc typographique nous fait entendre les échos des maux du poète. Il devient une harmonisation entre la vue et l'ouïe. Il incite le lecteur à recevoir une certaine émotion, il impose des orientations bien tentées du poète. Les pauses conscientes du poète imposent une accentuation de quelques valeurs et de quelques signifiés pour valoriser leur compréhension. Le blanc devient l'équivalent doux et silencieux de ce qui est interdit de l'annoncer.

Chez Damas, le rythme qui domine est ce qu'on désigne par le "rythme cardiaque" (Hernandez-Monmarty 2019, 33), c'est-à-dire un rythme qui suit les émotions, leur perturbation et les battements du cœur. Le poème devient une gravure de l'intimité abstraite que l'on peut recevoir visuellement, un rythme qui met en évidence l'harmonisation corps-langage. Chez Damas, le corps, par ses signes et son mouvement soit vibrant ou dansant, encadre un langage implicite.

Les formes irrégulières des vers et la disposition surprenante du blanc valorisent une coupure rythmique s'adaptant chez le poète avec le chaos qu'il éprouve: conflit des peaux, discrimination, racisme, oppression, éclatement de la guerre, désordre, chagrin et malheur. Elles représentent une révolution sur la forme régulière qui représente, d'après lui, le régime colonisateur opresseur et par conséquent sur la réalité actuelle. Cette forme du poème déchiré par l'intervention du blanc s'adapte avec un monde agité, morcelé par les guerres et gonflant d'asymétries et de conflits secteurs. La disposition du blanc est le reflet des sentiments perturbés du poète envers un monde agité.

Le blanc typographique met en valeur le labyrinthe frappant psychologique et spatial qu'éprouve le poète en vue de son déracinement de sa terre natale. Il traduit un besoin incessant de retour vers sa terre natale. Le blanc est le reflet d'un dépaysement sur le niveau mental et spatial.La disposition du blanc typographique s'adapte avec l'armature rythmique dans "Pigments" 
évoquant le rythme du jazz, du tam-tam, du tambour et d'autres musiques valorisant le layrinthe et la diaspora nègre.

Aussi la disposition du blanc typographique s'adapte-t-elle avec une volonté intime chez Damas: la preuve de la supériorité raciale nègre. Dans sa conception poétique, le blanc, le vide ou la forme représentent l'Homme blanc, vaniteux de la fausse croyance de sa civilisation et de sa distinction. Le noir, l'encre, les mots ou le fond et l'essence, désignent l'Homme Noir. Le blanc typoghraphique se forme en suivant la disposition et l'itinéraire des mots ou du noir. Donc, d'après lui, le maître est le noir pas le blanc.

Ainsi, Dans "Pigments", Damas met-il en évidence le paratexte et la typographie engendrant un langage ou une communication implicite bien touchants et bien motivants.

\section{Références}

\section{I- $\quad$ Corpus de l'étude:}

- Damas, L.G (2001), Pigments - Névralgies, Editios établie et postfacée par Sandrine Poujols, Paris, Présence Africaine.

II- Ouvrages consacrés à la Négritude et partiellemment à LéonGontran Damas

- Abiola Irele, F. (2008), Négritude et condition africaine, Paris, Karthala.

- Biasini, E. (1995), Grands travaux: de l'Afrique au Louvre, Paris, Editions Odile Jacob.

- Chancé, D. (2001), Poétique baroque de la Caraïbe, Paris, Khartala.

- Cheymol, M et Ollé-Laprun, Ph. ( 2010), Aimé Césaire à l'auvre: actes $d u$ colloque international, Paris, Editions des Archives Contemporaines.

- Constant, I. et Mabana, KC. (2009), Negritude: Legacy and Present Relevance, Londres, Cambridge Scholars, 2009

- Corre, L. (2017), Les échos de la poésie de la Négritude dans le rab français des années 1980 á 2017, Université Paris Ouest Nanterre, la Défense. 
- Dieng, A A. (2009), Les étudiants africains et la litterature negroafricaine d'expression francaise, North West Region, Cameroun

- Dehoux, A. (2018), Centres et périphéries de la littérature mondiale: une pensée connectée de la diversitée, Paris, Connaissances et Savoirs, Dépôt Légal

- Durpaire, F. (2006), France blanche, Colère noire, Paris, Odile Jacob.

- $\quad$ Fanon, F. (1952), Peau noire masques blancs, Paris, Seuil.

- Hernandez-Monmarty, E.(2019), Musicalité, corps et spiritualité dans la poésie de la négritude chez Césaire, Senghor et Craveirinha, Centre Transdisciplinaire de la Littérature, Université Côte d'Azure, https://tel.archives-ouvertes.fr/tel-02094265

- Huannou, A (1984), La littérature béninoise de langue francaise: des origines à nos jours, Paris, Kharthala.

- Jacques-Raphaël, G. (2004), Cacoïsme littéraire: la fonction du personnage américain dans le roman haïtien à partir de 1915, Bruxelles, Peter Lang.

- Leiner, J. (1993), Aimé Césaire: le terreau primordial, Volume 1, Dischingerweg, Cunter Narr Verlag Tübingen, Etudes Littéraires Françaises.

- Malela Buata, B. (2008), Les écrivains afro-antillais à Paris (19201960): Stratégies et postures identaires, Paris, Khartala.

- $\quad$ Mansfield, E. (2009), La symbolique du regard: regardants et regardés dans la poésie antillaise d'expression francaise, Saint-Denis, Publibook, coll. "Lettres \& Langues, Lettres Modernes

- Moudileno, L. (1997), L'écrivain antillais au miroir de sa littérature: mises en scène et mise en abyme du roman antillais, Paris, Khartala.

- Quaghebeur, M. (2008), Analyse et enseignement des littératures francophones: tentatives, réticences, responsabilitées, Bruxuelles, Peter Lang. 
- Rabindrânâth Tagore et Neruda, P. (2011), Aimé Césaire: pour un universel réconcilié, Paris, Unesco, 2011

- $\quad$ Saura, C-P. (2016), Surréalisme et Négritude: de Breton à Senghor et Lorca, Paris, Sorbonne-Madride.

- Senghor, L.S (1971), Liberté II, Négritude et Humanisme, Paris, Seuil,

- Soubrier, V. (2010), Le Théâtre de Koffi Kwahulé: L'utopie d'une écriture-jazz, Amesterdam, Rodopi.

- Talahite-Moodley, A. (2007), Problématiques identitaires et discours de l'exil dans les littératures francophones, Ottawa, les Presses d'université d'Ottawa,

- Taiana, C et Ruprecht, A. (1995), Reordering of Culture: Latin America, the Caribbean and Canada in the Hood, Carleton University Press.

- Thomas, C. et Lecomte, M (1983), Le Facteur ethnique aux ÉtatsUnis et au Canada, Presses Univ. Septentrion, Université de Lille III.

- - Toh Bi, E. (2016), Les ingrédiens du Lyrisme dans la poésie Négritudienne, Université de Bouaké, Côte d'Ivoire, , www.pandesmuses.fr > negritudienne

III- Articles consacrés à la Négritude et aux poètes négro-africains

- Achille, A., Contre le colonialisme, https://www.reseaucanope.fr/tdc/tous-les-numeros/lengagement-

- Aquila, (1986), Chestnut Hill Studies, in_Modern Languages and Literatures, Cartier, N.R. (Ed.).

- Boyce-Davies. C, et Ogundipe, M. (1995), Black Women's Diasporas, in Moving Beyond Boundaries (Vol. 2): New York University Press, Carole Boyce Davies.

- Cabanis, J.Ch. (2018), Le Pain de Dieu, le Corps du Christ, rend présent le Christ au monde, oraweb.net > le-pain-de-vie-le-corps-...

- Césaire, A. (2013), Nègreries : jeunesse noire et assimilation, in Les Temps Modernes, $\underline{\left(n^{\circ} 676\right)}$, pp 246 à 248 , www.cairn.info > revue-lestemps-m... 
- Chanda, T. (2013), Aimé Césaire et le mouvement de la Négritude,http://www.rfi.fr/fr/tirthankar-chanda.

- $\quad$ - Chouak, Y. (2018), Christiane Yandé Diop, une Présence Africaine, www.rfi.fr $>$... $>$ in sol majeur,,$\left(\mathbf{n}^{\circ} \mathbf{2 4 1}\right)$, pages 43 à 53

- Combe, D. (2014), Le Noir et le langage, Fanon eT Césaire S, in Collège international de Philosophie, $\mathrm{n}^{\circ} \mathbf{8 3}, \quad \mathrm{pp} .11 \quad-\quad 21$, https://www.cairn.info/revue-rue-descartes-2014-4-page-11.htm

- Diaw, A. (2010), Du fusil à A la Plume, la valorisation de l'amitié e de l'amour dans Hosties Noires de Léopold Sédar Senghor, in Ethiopiques n ${ }^{\circ} 4$. , Littérature, philosophie et 1er semestre , http://ethiopiques.refer.sn

- Dieng, A. A (2009), Les etudiants africains et la litterature negroafricaine d'expression francaise, Published by African Books, https://muse.jhu.edu/book/16927

- $\quad$ Delas, D. (2017), Afriques en poésie, apela.hypotheses.org > ...

- $\quad$ Kesteloot, L. (2012), La littérature négro-africaine face à l'histoire de l'Afrique, in Afrique contemporaine

- Jonassaint, J. (2013), Francophonies d'Amérique Césaire et Haïti, des apports à évaluer, in Engagement social et communautés d'allégeance, Numéro 36, Les Presses de l'Université d'Ottawa, Centre de recherche en civilisation canadienne-française https://id.erudit.org/iderudit/1029380arDOI ISSN1183-2487

- Labrune-Badiane, C. (2013), Voyages vers un « continent imaginaire »: Antillais au Sénégal (1960-1970), in Outre-Mers, Revue d'histoire , pp. 137-149 , Fait partie d'un numéro thématique: Les territoires de l'histoire antillaise 
- Lepidi, P. (2019), Tirailleurs sénégalais : «On les a forcés à s’engager, et aujourd'hui on laisse leurs petits-fils se noyer dans la Méditerranée, www.lemonde.fr > Afrique > Histoire

- $\quad$ Magnier, B (1985), Entretien avec Birago Diop, Intersections littéraires d'Afrique et des Caraïbes, Propos recueillis en Bernard et Publiés dans la revue " Notre Librairie », ${ }^{\circ} 81$, www.littafcar.org > actualites > entret...

- Manchuelle, F. (1992), Le rôle des Antillais dans l'apparition du nationalisme culturel en Afrique noire francophone, in Cahiers d'Études africaines, No. 127, pp. 375-408, persée

- Misuzu, T. (2009), La pensée culturelle de Senghor: la symbiose des langues et des cultures, in La Societe Japonaise de Didactique du Français,https://www.jstage.jst.go.jp/article/rjdf/4/2/4_KJ00009937192/_pdf

- Müller, E.W. (1996), L'Etudiant Noir, négritude et racisme. in Critique d'une critique, Anthropos, pp. 5-18, Published by: Nomos Verlagsgesellschaft mbH, https://www.jstor.org/stable/40465269

- Ngwe, R. (2016), Poésie africaine et écriture de l'histoire, In Pour la poésie, pp 237-258, www.cairn.info > pour-la-poesie--97...

- Proteau, L. (2001), Entre poetique et politique aime cesaire et la «negritude», in Sociétés contemporaines 2001/4 ( $\mathbf{n}^{\mathbf{0}}$ 44), pages 15 à 39, www.cairn.info > revue-diogene-201...

- Sagadou, J.P (2019), La négritud de Dieu, https://africa.lacroix.com/contribution \% E2\% 80\%89-la-negritude-dedieu \% E2\%80\%89/

- $\quad$ Tardy Joubert, H.(2014), Sartre et la Négritude : de l'existence à l'histoire, $\left(\mathrm{n}^{\circ} 83\right)$, pp 36 - 49 , www.cairn.info > revue-rue-descartes...

- Toumson, R. (2003), Les littératures caribéennes francophones. Problèmes et Perspectives, in Cahiers de l'Association internationale des études francaises, no. $\underline{55}$ pp. 103-121. Persée, www.persee.fr , caief_0571-5865_2003 num 55_1_1488

- Treffel, F. (2014), Parlers africains d'hier et d'au jourd'hui: de la Négritude à la Néo-Gritude, in Éla. Études de linguistique appliquée, $\mathbf{n}^{\circ}$ 176 , 395-406, Klincksieck https://www.cairn.info/revue-ela-2014-4page-395.htm. 
- W. Scheel, Ch.

- (2016), Henri Jean-Louis Baghio'o (2016), Île en île, ile-en-ile.org , baghio_henri_jean-lo...

- (2017), Jean-Louis Baghio'o père et fils, Deux écrivains antillais du vingtième siècle entre quatre continents, www.cairn.info > article

IV- Ouvrages consacrés entièrement à Léon-Gontran Damas

- $\quad$ Lapierre, J. (2016), La communication: de l'écart dans Pigments suivi de Névralgies de Léon-Gontran Damas, Quebec Canada, Université de Laval.

- Vete-Congolo, H. (2015), Léon-Gontran damas, Une Négritude entière, Paris, L'Harmaton.

V- $\quad$ Articles consacrés à Léon-Gontran Damas

0) Bergeret, Y. (2007), Pigments de Léon Gontran Damas, l'histoire par l'image, histoire-image.org > etudes > pigmen...

1) Chalendar, P. et Chalendar, G. (1982), Le Vécu Nègre, une lecture de «Pigment» de Léon Gontran Damas, in Ethiopiques numéro 30 revue socialiste de culture négro-africaine $2 \mathrm{e}$ trimestre, http://ethiopiques.refer.sn

2) De Groof, M. et Gyssels, K. (2015), “Give Me Back My Black Dolls: Damas' Africa and Its Musei $\square$ cation, in Journal of Cross_Cultural Image Studies, Revue d'Études Interculturelles de l'Image From Poetry , IMAGINATIONS, http://imaginations.csj.ualberta.ca

3) Fermi, E. (2016), Léon-Gontran Damas. Cent ans en noir et blanc, éd. Antonella Emina, journals.openedition.org > studifranc...

Ferrarini, H. (2013), Léon-Gontrant Damas, l'étrange choix Poétique de Christiane Taubira, http://www.slate.fr/story/67853/etrange-choixpoetique-de-christiane-taubira

4) Gyssels, K.

5) (2010), La poésie par le théâtre de Gontran Damas, africultures.com , la-poesie-par-le-th...

6) (2009), Léon-Gontran Damas et le mythe de l'Améridien, in Dalhousie French Studies, Vol. 86, Littératures francophones: Mythes et exotismes à l'ère de la mondialisation, pp. 45-56, Published by: Dalhousie University, https:// 
7) (2017), Guy Tirolien et Léon G. Damas : deux antillectuels mémorables, la-plume-francophone.com > guy-tir...

8) Latidine, Y. (2009), La représentation de la femme dans l'œuvre de Léon-Gontran Damas, http://ile-en-ile.org/yasminah-latidine-la-femmedans-loeuvre-de-damas/

9) Lubabu M.K, T. (2012), Léon-Gontran Damas, le troisième homme, https://www.jeuneafrique.com/142176/culture/n-gritude-l-on-gontrandamas-le-troisi-me-homme/

- Macé, M. (2008), Léon Gontran Damas, in L'Arbre à Palabres, $\mathrm{N}^{\circ}$ Spécial, , www.fabula.org / actualites > l-arbre...

10) Nirelep , H. (2015), Court hommage à Léon-Gontran DAMAS, https://blogs.mediapart.fr/harry-nirelep/blog/280315/court-hommageleon-gontran-damas.

11) Saïd, G. (2016), Léon-Gontran Damas : corps colonisé, poésie mise à nu, in DIACRITIK, https://diacritik.com/2016/12/21/leon-gontrandamas-corps-colonise-poesie-mise-a-nu/

12) Triay, Ph.(2016), Une biographie de Léon-Gontran Damas à l'intention des jeunes, lalere.francetvinfo.fr > biographie-le...

13) Vété-Congolo, $\mathrm{H}$.

- ( 2014), La poésie explicative de Damas: de Pigments à Dernière escale, pp 207-215, Published online, www.tandfonline.com >abs >

14) (2014), Léon-gontrant Damas: Couper du fer qui coupe...les fers ou Ecrire au Coutelas Damas, L'Amour et la Justice, Etthiopiques no.92, ${ }^{\mathrm{er}}$ semestre, http://ethiopiques.refer.sn/spip.php?page=imprimerarticle\&id_article $=1905$

VI- Ouvrages consacrés à la poésie

15) Briolet, D. (1995) Lire la poésie du XXe siècle, Paris, Dunod.

16) Méniel, B. (2004), Renaissance de l'épopée: la poésie épique en France de 1572 à 1623, Genève, Droz

17) Castin, N. (1998), Sens et sensibilité en poésie moderne et contemporaine, Paris, PUF. 
18) Décaudin, M. (2013), La crise des valeurs symbolistes: vingt ans de poésie française, 1895-1914, Paris, Champion classiques.

19) Labarthe, J. (2004), Formes modernes de la poésie épique: nouvelles approche, nouvelle Poétique comparatiste, Bruxuelles, Peter Lang.

- Broghy, M et Gallaghar, M. (2006), Sens et présence du sujet poétique: la poésie de la France et du monde francophone depuis 1980, Amesterdam, Rodopi.

- $\quad$ Riffaterre, M. (1983), Sémiotique de la poésie, Paris. Seuil

- $\quad$ Rodríguez, A. (2003), Le pacte lyrique: configuration discursive et interaction affective, Sprimont, Belgique, Margada.

VII- Articles consacrés à la poésie

0) Acone, L. (2017), Danser la Rédemption, in Dante e l'arte 4, 2017. 101-124, Laboratoire de Médiévistique Occidentale de Paris, Université Paris 1 Panthéon Sorbonneludmila.acone@yahoo.fr

1) Foucaud, V. (2009), La poésie visuelle : essai de definition, France, Pessac,. https://hal.archives-ouvertes.fr/hal-00658808

2) Boulogne, M. ( 2019), Poésie: éditions à dos d'âne, courts récits biographiques, http://ww2.ac-poitiers.fr/doc/spip.php?article786

3) Foucaud, V. (2009), La poésie visuelle : essai de définition, FranceParis, Pessac, France. https://hal.archives-ouvertes.fr/hal-00658808

4) Radeljković, I. (2015), Les débuts de la poésie visuelle, University of Sarajevo, www.researchgate.net.

VIII- Ouvrages consacrés à la linguistique et à la stylistique

3 Amon, E. et Bomati Y.:

- (1993), Commentaire du texte, Paris, Larousse.

-(1994), Vocabulaire pour la dissertation, Paris, Larousse.

- $\quad$ Barthes, R, (1957), Mythologies, PARIS, Seuil.

- Calas, F. (2007), Le texte didascalique à l'épreuve de la lecture et de la representation, Presses Universitaires de Bordeaux, Pesace,

- Calas F. et Charbonneau D-R (2000), Méthode du commentaire stylistique, Paris, Nathan.

- Cressot M. (1976), Le Style et ses techniques, Précis d'analyse stylistique, Paris, PUF. 
- Dessons, G.(1991) Introduction à l'analyse du poème, Paris, Bordas.

- Dubois, J. (1994). Dictionnaire de Linguistique et des Sciences du Langage, Paris, Larousse.

- Fournier, J. ( 2003), Scénarisation et multimédia: processus de scénarisation interactive, Les Presses de L'université Laval, 2003

- $\quad$ Fromilhague ( C.) et Sancier ( A.):

- $\quad$ (1991), Introduction à l'analyse stylistique, Paris, Bordas.

- $\quad$ (1999), Analyses stylistiques, Formes et genres, Paris, Dunod.

- $\quad$ Gardes-Tamine, J. (2001), La stylistique, Paris Armand Colin.

- $\quad$ Gicquel, B. (1999), Stylistique littéraire et informatique, Artois Presses Université,

- Grammont (M. (1965), Petit Traité de versification française, Paris, Armand Colin

- $\quad$ Mével, P.A et Tattam, H (2010), Language Et Ses Contexts, Bruxuelles-Bern, Peter Lang.

- $\quad$ Molinié, G. (1986), Elements de stylistique francaise, Paris, PUF. PUF.

Molinié, G. et Chané, P. (1994), Qu'est-ce qu'un style?, Paris,

- $\quad$ Rey-Debove J.(1978), Le Métalangage, Etude linguistique sur le langage, Paris, Le Robert.

- $\quad$ Salbayre, S. et Vincent-Arnaud, N. (2006), L'analyse stylistique: textes littéraires de langue anglaise, Toulouse, Presses universitaires de Mirail.

IX- Articles consacrés à la stylistique

- Lipsky, A. (2003), Pour une description sémantique et morphosyntaxique du participe francais et allemand, in Langages, No. 149, pp. 71-85, Fait partie d'un numéro thématique : Participe présent et gérondif , Persée.

X- Ouvrages consacacrés au paratexte, à la typographie et à la ponctuation

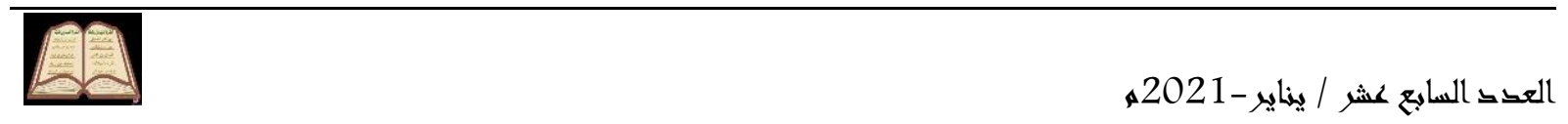


- Arabyan, M. (1994), Le Paragraphe narratif, Etude typographique de la ponctuation textuelle dans les récits classiques et modernes, Paris, Harmaton.

- Adam, J.M. (2011), Les Textes: Types et Prototypes, Paris, Armand Colin.

- Adam, J-M. et Bonhomme, M. (2011), L'argumentation publicitaire: Rhétorique de l'éloge et de lapersuasion, Paris, Armand Colin.

- Catach, N. (1994), La Ponctuation, Histoire et système, Que sais-je?, Paris, PUF.

- H.Hoek, L. (1981), La marque du titre, dispositifs sémiotiques d'une pratique textuelle, Paris, Mouton Editeur.

- Mactoux,M M et Geny, E (1990), Mélanges Pierre Lévêque: Anthropologie et société, Centre de recherché d'Histoire Ancienne, Volume 101,Paris, Presses Univ. Franche-Comté

- Milliot, V. (1995), Les "Cris de Paris", ou, Le peuple travesti: les représentations des petits métiers parisiens (XVIe-XVIIIe siècles), Paris, Publications de la Sorbonne.

- Cayuela, A. (1996), Le Paratexte au siècle d'or: prose romanesque, livres et lecteurs en Espagne au XVIIe siècle, Genève, Droz.

- GENETTE, G. (1987), Seuils, Paris, Seuil.

- Joly, M. (2015). Introduction à l'analyse de l'image, Paris, Armand Colin.

- Leroy,S. (2004), Le nom propre en français, Paris, Ophrys.

- Ribière, M. et Baeten, J. (1994), Temps, Narration et Image Fixe, Nietherlands, Les auteurs des articles et les rédacteur

- Starobinski, J. (1978), "Préface", Pour une esthétique de la réception, Paris, Gallimard, coll. "TEL".

XI- Articles consacrés au paratexte, à la typographie et à la

\section{ponctuation}

- Amrit, H. (1995), Les stratégies paratextuelles dans l'œuvre de Réjean Ducharme, in Annales Littéraires de l'université de Besancon, no 554, Paris, Les Belles Lettres.

- Bokiba, A.P (2006), Le Paratexte dans la littératureafricaine francophone: Léopold Sédar Senghor et Henri Lopes.Paris-Budapest-Kinshasa-TorinoOuagadougou, in Études littéraires africaines, Paris, L'Harmattan, Numéro 22, 
https://id.erudit.org/iderudit/1041258arDOI https://doi.org/10.7202/1041258ar, Association

- Carrara, M.A (2012), L'incipit : une première phrase essentielle, www.aproposdecriture.com > lincipit-une-premiere-phr.

- Formentelli, E. (1977). Présence du blanc, Figures du moins, in L'Espace et la lettre, Coll.dirigée par Bourgois ( Ch.), Paris, Bordas

- Lane, Ph. (1991), Seuils éditoriaux, in Espace Temps,_pp. 91-108, Fait partie d'un numéro thématique : La fabrique des sciences sociales. Lectures d'une écriture.

- Le Vez,Y ( 2013), Approche sémiologique de l'image, unmondemoderne.wordpress.com > ...

- Meylan, J.P. (1970), Les Expressionnistes allemands et la littérature francaise, in Die Aktion, Etudes littéraires, Les relations littéraires francoallemandes au XXe siècle, Département des littératures de l'Université Laval, Volume 3, numéro 3, pp 303-328. https://doi.org/10.7202/500145ar. - Moricheau-Airaud, B. (2017), Pour une analyse doublement critique de l'illustration de la première de couverture, Département de Lettres Classiques et Modernes, Centre de Recherche en Poétique, in Histoire Littéraire et en Linguistique, Université de Pau et des Pays de l'Adour.

- Purnelle, G. (2005), Pour une description typographique du poème, orbi.uliege.be , bitstream , Purnelle13.

Roy, M. (2008), Du titre littéraire et de ses effets de lecture, in Protée, Volume 36, Numéro 3, hiver 2008, p. 47-56, https://id.erudit.org/iderudit/019633ar adresse copiéeune erreur s'est produite

- Saminadayar-Perrin, C. (2-15), L'effet-titre, www.medias19.org

- TISSIER-DESBORDES, E. (2004), L'analyse de visuels: Pour une complémentarité des principales approaches, in Décisions Marketing, No. 36, (Octobre-Décembre 2004), pp. 63-74, in Association Française du Marketing, https://www.jstor.org/stable/40593001.

- Véron, E. (1994), De L'image sémiologique aux Discursivités, le temps d'une photo, Université de Paris, www.cairn.info > revue-hermes-la-re.

- Zao, A O. (2016), La signification cachée des couleurs en communication visuelle, evolutiongraphique.com > la-signification-cachee-des-c... 
XII- Sites généraux consultés

a) "Léon-gontrant Damas", https://migrateurs-transatlantique.pagespersoorange.fr/L-G_Damas.html, consulté le 5 janvier 2020.

b) "Analyse de S.O.S (Damas)", https://www.etudier.com/dissertations/AnalyseDe-s-o-s-Damas/50841632.html, consulté le 9 janvier.

- "Léon Gontran Damas, entre fureur et désenchantement", www.franceculture.fr > tire-ta-langue, consulté le 10 janvier 2020.

"Définition de la Négritude selon léon gontran", damasdistribuidoraapolo.com.br > journal > f... , consulté le 20 janvier 2020

c) "Définition de la négritude selon Léon-Gontan Damas", http://avazooterapia.org.br/journal/kgfil.php?5b3f03=d\% C3\%A9finitionde-la-n\% C3\% A9gritude-selon-1\% C3\% A9on-gontran-damas, consulté le 25 janvier 2020.

d) "Bob Boross , Histoire de la danse Jazz", www.offjazz.com, consulté le 10 février 2020.

e) "Le catholicisme a façonné nos repas", croire.la-croix.com > Vivre-enchretien diacritik.com >, consulté le 15 février 2020.

f) "Léon Damas | Étudiante éternelle, «Hoquet» Léon-Gontran Damas" Pigments, 1937", etudianteeternelle.wordpress.com > leon-dam, consulté le 15 mai 2020

g) "Entre poetique et politique aime cesaire et la « negritude - Cairn", www.cairn.info > revue-societes-cont, consulté le 25 mai 2020.

h) "Francophonies d'Amérique", https://apropos.erudit.org/fr/usagers/politiquedutilisation/, consulté le 30 mai 2020.

i) "Damas, L.G., Negritude,extrait de Hoquet 1939", yjohri.pagespersoorange.fr, consulté le 5 juin 2020.

- "Entre les Français et le pain, une histoire d'amour", www.observatoiredupain.fr > la-culture-pain-en-France, consulté le 8 juin 2020.

k) "Les couleurs dans les cultures : Quelles significations ?", www.targethnic.com, consulté le 10 juin 2020.

1) "Aimé Césairem Nègreries : jeunesse noire et assimilation", www.cairn.info > revue-les-temps-m.... consulté le 14 juin 2020.

- "Robert Desnos - Poètes en résistance", - Réseau Canopé, www.reseau-canope.fr > poetes $>$ rob..., consulté le 20 juin 2020. 
- "Robert Desnos, le "prince du surréalisme", alias "Robert le .... www.bulledemanou.com > 2015/03, consulté le 22 juin 2020.

- www.cnrtl.fr > definition > postface, consulté le 28 juin 2020.

- www.larousse.fr > francais > dédicace, consulté le 2juillet 2020.

p) $\underline{w w w . l a r o u s s e . f r}>$ francais $>$ tam-tam, consulté le 7 juillet 2020.

- $\quad$ fr.wikipedia.org > wiki > Asiatique, consulté le 15 juillet 2020.

q) fr.wikipedia.org > wiki > Gravure su..., consulté le 25 juillet 2020.

r) fr.wikipedia.org > wiki > Banjo, consulté le 28 juillet 2010.

s) https://doi.org/10.7202/1029380ar, consulté le 6 aôut. www.espacefrancais.com > la-preface, consulté le 10 octobre.

u) https://www.superprof.fr/ressources/langues/francais/lyceefr3/2nd-fr3/titre-extrait-information.html, consulté le 15 octobre. v) https://doi.org/10.7202/019633ar adresse copiéeune erreur s'est produite, consulté le 20 octobre.

w) data.bnf.fr > jacques howlett, consulté le 25 octobre 2020.

x) https://www.erudit.org/fr/, consulté le 27 octbre 2020.

y) https://la1ere.francetvinfo.fr/biographie-leon-gontran-damas-intention-jeunes-407565.html, consulté le 29 octobre 2020.

z) www.jstor.org/stable/40838029, consulté le 30 octobre 2020. aa) documents.irevues.inist.fr > handle, consulté le 2 novembre 2020. 